\title{
Optimal Insurance with Rank-Dependent Utility and Increasing Indemnities
}

\author{
Zuo Quan $\mathrm{Xu}^{*} \quad$ Xun Yu Zhou ${ }^{\dagger} \quad$ Shengchao Zhuang ${ }^{\ddagger}$
}

October 11, 2018

\begin{abstract}
Bernard et al. (2015) study an optimal insurance design problem where an individual's preference is of the rank-dependent utility (RDU) type, and show that in general an optimal contract covers both large and small losses. However, their contracts suffer from a problem of moral hazard for paying more compensation for a smaller loss. This paper addresses this setback by exogenously imposing the constraint that both the indemnity function and the insured's retention function be increasing with respect to the loss. We characterize the optimal solutions via calculus of variations, and then apply the result to obtain explicitly expressed contracts for problems with Yaari's dual criterion and general RDU. Finally, we use a numerical example to compare the results between ours and that of Bernard et al. (2015).
\end{abstract}

Keywords: optimal insurance design, rank-dependent utility theory, Yaari's dual criterion, probability weighting function, moral hazard, indemnity function, retention function, quantile formulation.

*Department of Applied Mathematics, Hong Kong Polytechnic University, Kowloon, Hong Kong. Email: maxu@polyu.edu.hk. This author acknowledges financial supports from Hong Kong Early Career Scheme (No. 533112), Hong Kong General Research Fund (No. 529711) and NNSF of China (No. 11471276).

${ }^{\dagger}$ Mathematical Institute and Nomura Centre for Mathematical Finance, and Oxford-Man Institute of Quantitative Finance, The University of Oxford, Oxford OX2 6GG, UK. This author acknowledges supports from a start-up fund of the University of Oxford, and research grants from the Nomura Centre for Mathematical Finance and the Oxford-Man Institute of Quantitative Finance.

${ }_{\ddagger}^{\ddagger}$ Department of Statistics and Actuarial Science, University of Waterloo, Waterloo, Ontario, N2L 3G1, Canada. 


\section{Introduction}

Optimal insurance contract design is an important problem, manifested not only in theory but also in insurance and financial practices. The problem is to determine the optimal amount of compensation as a function of the loss - called indemnity - so as to maximize the insured's satisfaction, subject to the participation constraint of the insurer.

In the insurance literature, most of the work assume that the insurer is risk neutral while the insured is a risk-averse expected utility (EU) maximizer; see e.g. Arrow (1963), Raviv (1979), and Gollier and Schlesinger (1996). In this case, the optimal contract is in general a deductible one that covers part of the loss in excess of a deductible level.

However, the EU theory has received many criticisms, for it fails to explain numerous experimental observations and theoretical puzzles. In the context of insurance contracting, the classical EU-based models cannot explain some behaviors in insurance demand such as that for small losses (e.g. demand for warranties); see a detailed discussion in Bernard et al. (2015).

In order to overcome this drawback of the EU theory, different measures of evaluating uncertain outcomes have been put forward to depict human behaviors. A notable one is the rank-dependent utility (RDU) proposed by Quiggin (1982), which consists of a concave utility function and an inverse- $S$ shaped probability weighting (or distortion) function. 1 Through the probability weighting, the RDU theory captures the common observation that people tend to exaggerate small probabilities of extremely good and bad outcomes. With the development of advanced mathematical tools, the RDU preference has been applied to many areas of finance, including portfolio choice and option pricing. On the other hand, Barseghyan et al. (2013) use data on households' insurance deductible decisions in auto and home insurance to demonstrate the relevance and importance of the probability weighting and suggest the possibility of generalizing their conclusions to other insurance choices.

There have been also studies in the area of insurance contract design within the RDU framework; see for example Chateauneuf, Dana and Tallon (2000), Dana and Scarsini (2007), and Carlier and Dana (2008). However, all these papers assume that the probability weighting function is convex. Bernard et al. (2015) are probably the first to study RDU-based insurance contracting with inverse$S$ shaped weighting functions, using the quantile formulation originally developed for portfolio choice (Jin and Zhou 2008, He and Zhou 2011). They derive optimal contracts that not only insure large losses above a deductible level but also cover small ones. However, their contracts suffer from a severe

\footnotetext{
${ }^{1}$ The RDU preference reduces to Yaari's dual criterion (Yaari 1987) when the utility function is the identity one.
} 
problem of moral hazard, since they are not increasing with respect to the losses.2 As a consequence, insureds may be motivated to hide their true losses in order to obtain additional compensations; see a discussion on pp. 175-176 of Bernard et al. (2015).

This paper aims to address this setback. We consider the same insurance model as in Bernard et al. (2015), but adding an explicit constraint that both the indemnity function and the insured's retention function (i.e. the part of the losses to be born by the insured) must be globally increasing with respect to the losses. This constraint will rule out completely the aforementioned behaviour of moral hazard; yet mathematically it gives rise to substantial difficulty. The approach used in Bernard et al. (2015) no longer works. We develop a general approach to overcome this difficulty. Specifically, we first derive the necessary and sufficient conditions for optimal solutions via calculus of variations. Then we deduce explicitly expressed optimal contracts by a fine analysis on these conditions. An interesting finding is that, for a good and reasonable range of parameters specifications, there are only two types of optima contracts, one being the classical deductible one and the other a "three-fold" one covering both small and large losses.

The remainder of the paper is organized as follows. Section 2 presents the optimal insurance model under the RDU framework including its quantile formulation. Section 3 applies the calculus of variations to derive a general necessary and sufficient condition for optimal solutions. We then derive optimal contracts for Yaari's criterion and the general RDU in Sections 4 and 5, respectively. Section 6 provides a numerical example to illustrate our results. Finally, we conclude with Section 7. Proofs of some lemmas are placed in an Appendix.

\section{The Model}

In this section, we present the optimal insurance contracting model in which the insured has the RDU type of preferences, followed by its quantile formulation that will facilitate deriving the solutions.

\subsection{Problem formulation}

We follow Bernard et al. (2015) for the problem formulation except for one critical difference, which we will highlight. Let $(\Omega, \mathbf{F}, \mathbb{P})$ be a probability space. An insured, endowed with an initial wealth

\footnotetext{
${ }^{2}$ Throughout this paper, by an "increasing" function we mean a "non-decreasing" function, namely $f$ is increasing if $f(x) \geqslant f(y)$ whenever $x>y$. We say $f$ is "strictly increasing" if $f(x)>f(y)$ whenever $x>y$. Similar conventions are used for "decreasing" and "strictly decreasing" functions.
} 
$W_{0}$, faces a non-negative random loss $X$ supported in $[0, M]$, where $M$ is a given positive scalar. He chooses an insurance contract to protect himself from the loss, by paying a premium $\pi$ to the insurer in return for a compensation (or indemnity) in the case of a loss. This compensation is to be determined as a function of the loss $X$, denoted by $I(\cdot)$ throughout this paper. The retention function $R(X):=X-I(X)$ is thereby the part of the loss to be borne by the insured.

For a given $X$, the insured aims to choose an insurance contract that provides the best tradeoff between the premium and compensation based on his risk preference. In this paper, we consider the case when insured's preference is of the RDU type. This RDU preference consists of two components: a utility function $u: \mathbb{R}^{+} \mapsto \mathbb{R}^{+}$and a probability weighting function $T:[0,1] \mapsto[0,1]$. Let us denote by $V^{r d u}(W)$ the RDU of the final (random) wealth $W$ of an insured, which is a Choquet integral of $u(W)$ with respect to the capacity $T \circ \mathbb{P}$, i.e.,

$$
V^{r d u}(W)=\int u(W) d(T \circ \mathbb{P}):=\int_{\mathbb{R}^{+}} u(x) d\left[-T\left(1-F_{W}(x)\right)\right]
$$

where $F_{W}(\cdot)$ is the cumulative distribution function $(\mathrm{CDF})$ of $W$. Assuming that $T$ is differentiable, we can rewrite

$$
V^{r d u}(W)=\int_{\mathbb{R}^{+}} u(x) T^{\prime}\left(1-F_{W}(x)\right) d F_{W}(x) .
$$

If $T$ is inverse- $S$ shaped, that is, it is first concave and then convex; see Figure 1, then the above expression shows that the role $T$ plays is to overweigh both tails of $W$ when evaluating the mean of $u(W)$. On the other hand, if the insurer is risk-neutral and the cost of offering the compensation is proportional to the expectation of the indemnity, then the premium to be charged for an insurance contract should satisfy the participation constraint

$$
\pi \geqslant(1+\rho) E[I(X)]
$$

where the constant $\rho$ is the safety loading of the insurer.

It is natural to require an indemnity function to satisfy

$$
I(0)=0, \quad 0 \leqslant I(x) \leqslant x, \quad \forall 0 \leqslant x \leqslant M,
$$

a constraint that has been imposed in most insurance contracting literature. If the insured's preference is dictated by the classical EU theory, then the optimal contract is typically a deductible contract which automatically renders the indemnity function increasing; see e.g. Arrow (1971) and Raviv (1979). However, for the RDU preference the resulting optimal indemnity may not be an increasing function, as shown in Bernard et al. (2015). This may potentially cause moral hazard 
as pointed out earlier. Similarly, a non-monotone retention function may also lead to moral hazard. Consequently, to include the increasing constraint on the contract has been an outstanding open question.

In this paper, we require the indemnity function to satisfy $I(0)=0$ and $0 \leqslant I(x)-I(y) \leqslant$ $x-y, \forall 0 \leqslant y<x \leqslant M$. In other words, we constrain both indemnity and retention functions to be globally increasing.

We can now formulate our insurance contracting problem as

$$
\begin{array}{ll}
\max _{I(\cdot)} & V^{r d u}\left(W_{0}-\pi-X+I(X)\right) \\
\text { s.t. } & (1+\rho) E[I(X)] \leqslant \pi \\
& I(\cdot) \in \mathbb{I}
\end{array}
$$

where

$$
\mathbb{I}:=\{I(\cdot): I(0)=0,0 \leqslant I(x)-I(y) \leqslant x-y, \forall 0 \leqslant y \leqslant x \leqslant M\},
$$

and $W_{0}$ and $\pi$ are fixed scalars.

For any random variable $Y \geqslant 0$ a.s., define the quantile function of $Y$ as

$$
F_{Y}^{-1}(t):=\inf \left\{x \in \mathbb{R}^{+}: P(Y \leqslant x) \geqslant t\right\}, \quad t \in[0,1] .
$$

Note that any quantile function is nonnegative, increasing and left-continuous (ILC).

We now introduce the following assumptions that will be used hereafter.

Assumption 2.1 The random loss $X$ has a strictly increasing distribution function $F_{X}$. Moreover, $F_{X}^{-1}$ is absolutely continuous on $[0,1]$.

Assumption 2.2 (Concave Utility) The utility function $u: \mathbb{R}^{+} \mapsto \mathbb{R}^{+}$is strictly increasing and continuously differentiable. Furthermore, $u^{\prime}$ is decreasing.

Assumption 2.3 (Inverse-S Shaped Weighting) The probability weighting function $T$ is a continuous and strictly increasing mapping from [0,1] onto [0,1] and twice differentiable on (0,1). Moreover, there exists $b \in(0,1)$ such that $T^{\prime}(\cdot)$ is strictly decreasing on $(0, b)$ and strictly increasing on $(b, 1)$. Furthermore, $T^{\prime}(0+):=\lim _{z \downarrow 0} T^{\prime}(z)>1$ and $T^{\prime}(1-):=\lim _{z \uparrow 1} T^{\prime}(z)=+\infty$.

The first part of Assumption 2.1, crucial for the quantile formulation, is standard; see e.g. Raviv (1979). Note a significant difference from Bernard et al. (2015) is that here we allow $X$ to have 
atoms (which is usually the case in the insurance context). For example, let $X$ be distributed with $F_{X}(x)=\frac{1-\gamma e^{-\eta x}}{1-\gamma e^{-\eta M}}$ for $x \in[0, M]$, where $\gamma \in(0,1)$ and $\eta>0$. Then, $X$ satisfies Assumption 2.1, and has an atom at 0 with the probability $\mathbb{P}(X=0)=\frac{1-\gamma}{1-\gamma e^{-\eta M}}>0$. This assumption also ensures that $F_{X}^{-1}\left(F_{X}(x)\right) \equiv x, \forall x \in[0, M]$, a fact that will be used often in the subsequent analysis. Next, Assumption 2.2 is standard for a utility function. Finally, Assumption 2.3 is satisfied for many weighting functions proposed or used in the literature, e.g. that proposed by Tversky and Kahneman (1992) (parameterized by $\theta)$ :

$$
T_{\theta}(x)=\frac{x^{\theta}}{\left(x^{\theta}+(1-x)^{\theta}\right)^{\frac{1}{\theta}}} .
$$

Figure 1 displays this (inverse- $S$ shaped) weighting function (in blue) when $\theta=0.5$.

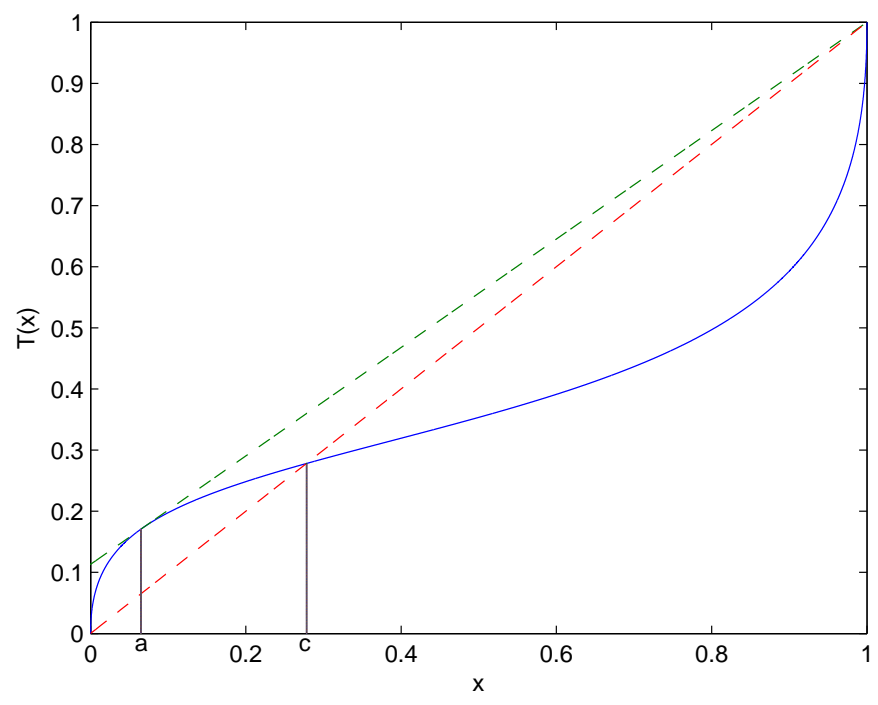

Figure 1: An inverse- $S$ shaped weighting function satisfying Assumption 2.3. The marked points $a$ and $c$ will be explained later.

In practice, most of the insurance contracts are not tailor-made for individual customers. Instead, an insurance company usually has contracts with different premiums to accommodate customers with different needs. Each contract is designed with the best interest of a representative customer in mind so as to stay marketable and competitive, while maintaining the desired profitability (the participation constraint). An insured can then choose one from the menu of contracts to cater for individual needs. Problem (22) is therefore motivated by the insurer's making of this menu.

If the premium $\pi \geqslant(1+\rho) E[X]$, then $I^{*}(x) \equiv x$ (corresponding to a full coverage) is feasible and maximizes the objective function in Problem (2) pointwisely; hence optimal. To rule out this 
trivial case, henceforth we restrict $0<\pi<(1+\rho) E[X]$. Moreover, we assume

$$
W_{0}-(1+\rho) E[X]-M \geqslant 0
$$

to ensure that the policyholder will not go bankrupt. This is because $W_{0}-\pi-M>0, \forall \pi \in$ $(0,(1+\rho) E[X])$.

It is more convenient to consider the retention function $R(x)=x-I(x)$ instead of $I(x)$ in our study below. Letting $\Delta:=E[X]-\frac{\pi}{1+\rho} \in(0, E[X]), W:=W_{0}-(1+\rho) E[X]>0, W_{\Delta}:=$ $W+(1+\rho) \Delta \equiv W_{0}-\pi$, we can reformulate (2) in terms of $R(\cdot)$ :

$$
\begin{array}{ll}
\max _{R(\cdot)} & V^{r d u}\left(W_{\Delta}-R(X)\right) \\
\text { s.t. } & E[R(X)] \geqslant \Delta, \\
& R(\cdot) \in \mathcal{R},
\end{array}
$$

where

$$
\mathcal{R}:=\{R(\cdot): R(0)=0,0 \leqslant R(x)-R(y) \leqslant x-y, \forall 0 \leqslant y \leqslant x \leqslant M\} .
$$

\subsection{Quantile Formulation}

The objective function in (5) is not concave in $R(X)$ (due to the nonlinear weighting function $T$ ), leading to a major difficulty in solving (5). However, under Assumption 2.3, we have

$$
\begin{aligned}
V^{r d u}\left(W_{\Delta}-R(X)\right) & =\int_{\mathbb{R}^{+}} u(x) d\left[-T\left(1-F_{W_{\Delta}-R(X)}(x)\right)\right] \\
& =\int_{0}^{1} u\left(F_{W_{\Delta}-R(X)}^{-1}(z)\right) T^{\prime}(1-z) d z=\int_{0}^{1} u\left(W_{\Delta}-F_{R(X)}^{-1}(1-z)\right) T^{\prime}(1-z) d z \\
& =\int_{0}^{1} u\left(W_{\Delta}-F_{R(X)}^{-1}(z)\right) T^{\prime}(z) d z,
\end{aligned}
$$

where the third equality is because $F_{W_{\Delta}-R(X)}^{-1}(z)=W_{\Delta}-F_{R(X)}^{-1}(1-z)$ except for a countable set of $z$. On the other hand, $E[R(X)] \geqslant \Delta$ is equivalent to $\int_{0}^{1} F_{R(X)}^{-1}(z) d z \geqslant \Delta$.

The above suggests that we may change the decision variable from the random variable $R(X)$ to

its quantile function $F_{R(X)}^{-1}$, with which the objective function of (5) becomes concave and the first constraint is linear. It remains to rewrite the monotonicity constraint (represented by the constraint set $\mathcal{R}$ ) also in terms of $F_{R(X)}^{-1}$. To this end, the next lemma plays an important role.

Lemma 2.1 Under Assumption [2.1, for any given $R(\cdot) \in \mathcal{R}$, we have

$$
R(x)=F_{R(X)}^{-1}\left(F_{X}(x)\right), \forall x \in[0, M]
$$


ProOF: First, by the monotonicity of $R(\cdot)$, we have $\mathbb{P}(R(X) \leqslant R(x)) \geqslant \mathbb{P}(X \leqslant x)=F_{X}(x)$, so by the definition of $F_{R(X)}^{-1}\left(F_{X}(x)\right)$, we conclude that $F_{R(X)}^{-1}\left(F_{X}(x)\right) \leqslant R(x)$. It suffices to prove the reverse inequality. Consider two cases.

- $R(x)=0$ : In this case, we have $F_{R(X)}^{-1}\left(F_{X}(x)\right)=0$ as quantile functions are nonnegative.

- $R(x)>0$ : It suffices to prove that $\mathbb{P}(R(X) \leqslant z)<F_{X}(x)$ for any $z<R(x)$. Take $z_{1}$ such that $z<z_{1}<R(x)$. By the continuity and monotonicity of $R(\cdot)$, there exists $y$ such that $y<x$ and $R(y)=z_{1}$. Then, $\mathbb{P}(R(X) \leqslant z) \leqslant \mathbb{P}\left(R(X)<z_{1}\right)=\mathbb{P}(R(X)<R(y)) \leqslant \mathbb{P}(X \leqslant y)=F_{X}(y)<$ $F_{X}(x)$, where we have used the fact that $F_{X}$ is strictly increasing under Assumption 2.1 .

The claim is thus proved.

In view of the above results, we can rewrite (5) as the following problem, in which the decision variable is $F_{R(X)}^{-1}(\cdot)$ (denoted by $G(\cdot)$ for simplicity):

$$
\begin{aligned}
\max _{G(\cdot)} & \int_{0}^{1} u\left(W_{\Delta}-G(z)\right) T^{\prime}(z) d z, \\
\text { s.t. } & \int_{0}^{1} G(z) d z \geqslant \Delta, \\
& G(\cdot) \in \mathbb{G},
\end{aligned}
$$

where $\mathbb{G}:=\left\{F_{R(X)}^{-1}(\cdot): R(\cdot) \in \mathcal{R}\right\}$.

In the absence of an explicit expression the constraint set $\mathbb{G}$ is hard to deal with. The following result addresses this issue. Note the major technical difficulty arises from the possible existence of the atoms of $X$.

Lemma 2.2 Under Assumption 2.1, we have

$$
\mathbb{G}=\left\{G(\cdot): G(\cdot) \text { is absolutely continuous, } G(0)=0,0 \leqslant G^{\prime}(z) \leqslant\left(F_{X}^{-1}\right)^{\prime}(z) \text {, a.e. } z \in[0,1]\right\} \text {. }
$$

Proof: We denote the right hand side of (7) by $\mathbb{G}_{1}$. For any $G(\cdot) \in \mathbb{G}$, there exists $R(\cdot) \in \mathcal{R}$ such that $G(\cdot)=F_{R(X)}^{-1}(\cdot)$. For any $0 \leqslant b<a \leqslant 1$, define $\underline{a}=\inf \{x \in[0, M]: R(x)=G(a)\}$, $\bar{a}=\sup \{x \in[0, M]: R(x)=G(a)\}$, define $\underline{b}$ and $\bar{b}$ similarly. Let us show that $\underline{a} \leqslant F_{X}^{-1}(a) \leqslant \bar{a}$. In fact, by definition,

$$
\begin{aligned}
F_{X}^{-1}(a) & =\inf \left\{x \in \mathbb{R}^{+}: F_{X}(x) \geqslant a\right\} \geqslant \inf \left\{x \in \mathbb{R}^{+}: G\left(F_{X}(x)\right) \geqslant G(a)\right\} \\
& =\inf \left\{x \in \mathbb{R}^{+}: R(x) \geqslant G(a)\right\}=\underline{a} .
\end{aligned}
$$


Suppose $F_{X}^{-1}(a)-\varepsilon>\bar{a}$ for some $\varepsilon>0$. Then by monotonicity,

$$
G(a)=R(\bar{a})<R\left(F_{X}^{-1}(a)-\varepsilon\right)=G\left(F_{X}\left(F_{X}^{-1}(a)-\varepsilon\right)\right) \leqslant G(a)
$$

where we have used the fact that $F_{X}\left(F_{X}^{-1}(a)-\varepsilon\right)<a$ to get the last inequality. This leads to a contradiction; hence it must hold that $F_{X}^{-1}(a) \leqslant \bar{a}$. Similarly, we can prove $\underline{b} \leqslant F_{X}^{-1}(b) \leqslant \bar{b}$. Then we have

$$
0 \leqslant G(a)-G(b)=R(\underline{a})-R(\bar{b}) \leqslant \underline{a}-\bar{b} \leqslant F_{X}^{-1}(a)-F_{X}^{-1}(b) .
$$

This inequality shows that $G$ is absolutely continuous since $F_{X}^{-1}$ is an absolutely continuous function under Assumption 2.1. Furthermore, it also implies $\left.0 \leqslant G^{\prime}(z) \leqslant\left(F_{X}^{-1}\right)^{\prime}(z)\right)$, a.e. $z \in[0,1]$. So we have established that $\mathbb{G} \subseteq \mathbb{G}_{1}$.

To prove the reverse inclusion, take any $G(\cdot) \in \mathbb{G}_{1}$ and define $R(\cdot)=G\left(F_{X}(\cdot)\right)$. It follows from Assumption 2.1 that $0 \leqslant R(0)=G\left(F_{X}(0)\right)-G(0) \leqslant F_{X}^{-1}\left(F_{X}(0)\right)-F_{X}^{-1}(0)=0$ and $0 \leqslant R(a)-R(b)=$ $G\left(F_{X}(a)\right)-G\left(F_{X}(b)\right) \leqslant F_{X}^{-1}\left(F_{X}(a)\right)-F_{X}^{-1}\left(F_{X}(b)\right)=a-b \forall 0 \leqslant b<a \leqslant 1$. Hence $R(\cdot) \in \mathcal{R}$. It now suffices to show $G(a)=F_{R(X)}^{-1}(a)$ for any $0 \leqslant a \leqslant 1$. If $G(a)=0$, then $G(a) \leqslant F_{R(X)}^{-1}(a)$ holds. Otherwise, for any $s<G(a)$, there exists $y$ such that $s<R(y)=G\left(F_{X}(y)\right)<G(a)$ by the continuity of $R(\cdot)$. Then by the monotonicity of $R(\cdot)$ and $G(\cdot)$, we have

$$
\mathbb{P}(R(X) \leqslant s) \leqslant \mathbb{P}(R(X)<R(y)) \leqslant \mathbb{P}(X \leqslant y)=F_{X}(y)<a
$$

which means $G(a) \leqslant F_{R(X)}^{-1}(a)$. Using the same notation, $\bar{a}$, as above, and noting that $G(a)=$ $R(\bar{a})=G\left(F_{X}(\bar{a})\right)$, we have $a \leqslant F_{X}(\bar{a})$ by the definition of $\bar{a}$ and the continuity of $R(\cdot)$. Moreover, it follows from

$$
\mathbb{P}(R(X) \leqslant G(a))=\mathbb{P}(R(X) \leqslant R(\bar{a}))=\mathbb{P}(X \leqslant \bar{a})=F_{X}(\bar{a})
$$

that $F_{R(X)}^{-1}\left(F_{X}(\bar{a})\right) \leqslant G(a)$. Therefore,

$$
G(a) \leqslant F_{R(X)}^{-1}(a) \leqslant F_{R(X)}^{-1}\left(F_{X}(\bar{a})\right) \leqslant G(a)
$$

holds by monotonicity. The desired result follows.

To solve (6), we apply the Lagrange dual method to remove the constraint $\int_{0}^{1} G(z) d z-\Delta \geqslant 0$ and consider the following auxiliary problem:

$$
\begin{aligned}
\max _{G(\cdot)} & U_{\Delta}(\lambda, G(\cdot)):=\int_{0}^{1}\left[u\left(W_{\Delta}-G(z)\right) T^{\prime}(z)+\lambda G(z)\right] d z-\lambda \Delta, \\
\text { s.t. } & G(\cdot) \in \mathbb{G} .
\end{aligned}
$$


The existence of the optimal solutions to (6) and (8) (for each given $\lambda \in \mathbb{R}^{+}$) is established in Appendix B, while the uniqueness is straightforward when the utility function $u$ is strictly concave.

To derive the optimal solution to (66), we first solve (8) to obtain an optimal solution, denoted by $\widetilde{G}_{\lambda}(\cdot)$. Then we determine $\lambda^{*} \in \mathbb{R}^{+}$by binding the constraint $\int_{0}^{1} \widetilde{G}_{\lambda^{*}}(z) d z=\Delta$. A standard duality argument then deduces that $G^{*}(\cdot):=\widetilde{G}_{\lambda^{*}}(\cdot)$ is an optimal solution to (6). Finally, an optimal solution to (5) is given by $R^{*}(z)=G^{*}\left(F_{X}(z)\right) \forall z \in[0, M]$ and that to $(1)$ by $I^{*}(z)=z-R^{*}(z) \forall z \in[0, M]$.

So our problem boils down to solving (8) . However, in doing so the constraint that $0 \leqslant G^{\prime}(z) \leqslant$ $\left(F_{X}^{-1}\right)^{\prime}(z)$ in $\mathbb{G}$ poses the major difficulty compared with Bernard et al. (2015).

\section{Characterization of Solutions}

In this section, we derive a necessary and sufficient condition for a solution to be optimal to (8)). Assume $\widetilde{G}_{\lambda}(\cdot)$ solves (8) with a fixed $\lambda$. Let $G(\cdot) \in \mathbb{G}$ be arbitrary and fixed. For any $\varepsilon \in(0,1)$, set $G^{\epsilon}(\cdot)=(1-\epsilon) \widetilde{G}_{\lambda}(\cdot)+\epsilon G(\cdot)$. Then $G^{\epsilon}(\cdot) \in \mathbb{G}$. By the optimality of $\widetilde{G}_{\lambda}(\cdot)$ and the concavity of $u$, we have

$$
\begin{aligned}
& 0 \geqslant \frac{1}{\varepsilon}\left\{\int_{0}^{1}\left[u\left(W_{\Delta}-G^{\epsilon}(z)\right) T^{\prime}(z)+\lambda G^{\epsilon}(z)\right] d z-\int_{0}^{1}\left[u\left(W_{\Delta}-\widetilde{G}_{\lambda}(z)\right) T^{\prime}(z)+\lambda \widetilde{G}_{\lambda}(z)\right] d z\right\} \\
& =\frac{1}{\varepsilon}\left\{\int_{0}^{1}\left[\left(u\left(W_{\Delta}-G^{\epsilon}(z)\right)-u\left(W_{\Delta}-\widetilde{G}_{\lambda}(z)\right)\right) T^{\prime}(z)+\lambda\left(G^{\epsilon}(z)-\widetilde{G}_{\lambda}(z)\right)\right] d z\right\} \\
& \geqslant \frac{1}{\varepsilon}\left\{\int_{0}^{1}\left[\left(u^{\prime}\left(W_{\Delta}-G^{\epsilon}(z)\right)\right)\left(W_{\Delta}-G^{\epsilon}(z)-W_{\Delta}+\widetilde{G}_{\lambda}(z)\right) T^{\prime}(z)+\lambda\left(G^{\epsilon}(z)-\widetilde{G}_{\lambda}(z)\right)\right] d z\right\} \\
& \stackrel{\epsilon \downarrow 0}{\longrightarrow} \int_{0}^{1}\left[\left(u^{\prime}\left(W_{\Delta}-\widetilde{G}_{\lambda}(z)\right)\right)\left(\widetilde{G}_{\lambda}(z)-G(z)\right) T^{\prime}(z)+\lambda\left(G(z)-\widetilde{G}_{\lambda}(z)\right)\right] d z \\
& =\int_{0}^{1}\left[u^{\prime}\left(W_{\Delta}-\widetilde{G}_{\lambda}(z)\right) T^{\prime}(z)-\lambda\right]\left(\widetilde{G}_{\lambda}(z)-G(z)\right) d z .
\end{aligned}
$$

Define

$$
N_{\lambda}(z):=-\int_{z}^{1}\left[u^{\prime}\left(W_{\Delta}-\widetilde{G}_{\lambda}(t)\right) T^{\prime}(t)-\lambda\right] d t, \quad z \in[0,1] .
$$

Then (9) yields

$$
\begin{aligned}
0 & \geqslant \int_{0}^{1}\left[u^{\prime}\left(W_{\Delta}-\widetilde{G}_{\lambda}(z)\right) T^{\prime}(z)-\lambda\right]\left(\widetilde{G}_{\lambda}(z)-G(z)\right) d z=\int_{0}^{1} \int_{0}^{z}\left(\widetilde{G}_{\lambda}^{\prime}(t)-G^{\prime}(t)\right) d t d N_{\lambda}(z) \\
& =\int_{0}^{1} \int_{t}^{1}\left(\widetilde{G}_{\lambda}^{\prime}(t)-G^{\prime}(t)\right) d N_{\lambda}(z) d t=\int_{0}^{1} N_{\lambda}(t)\left(G^{\prime}(t)-\widetilde{G}_{\lambda}^{\prime}(t)\right) d t
\end{aligned}
$$

leading to

$$
\int_{0}^{1} N_{\lambda}(z) G^{\prime}(z) d z \leqslant \int_{0}^{1} N_{\lambda}(z) \widetilde{G}_{\lambda}^{\prime}(z) d z, \quad \forall G(\cdot) \in \mathbb{G} .
$$


In other words, $\widetilde{G}_{\lambda}^{\prime}(\cdot)$ maximizes $\int_{0}^{1} N_{\lambda}(z) G^{\prime}(z) d z$ over $G(\cdot) \in \mathbb{G}$. Therefore, a necessary condition for $\widetilde{G}_{\lambda}(\cdot)$ to be optimal for (묘 is

$$
\widetilde{G}_{\lambda}^{\prime}(z) \stackrel{\text { a.e. }}{=} \begin{cases}0, & \text { if } N_{\lambda}(z)=\int_{z}^{1}\left[\lambda-u^{\prime}\left(W_{\Delta}-\widetilde{G}_{\lambda}(t)\right) T^{\prime}(t)\right] d t<0, \\ \in\left[0,\left(F_{X}^{-1}\right)^{\prime}(z)\right], & \text { if } N_{\lambda}(z)=\int_{z}^{1}\left[\lambda-u^{\prime}\left(W_{\Delta}-\widetilde{G}_{\lambda}(t)\right) T^{\prime}(t)\right] d t=0, \\ \left(F_{X}^{-1}\right)^{\prime}(z), & \text { if } N_{\lambda}(z)=\int_{z}^{1}\left[\lambda-u^{\prime}\left(W_{\Delta}-\widetilde{G}_{\lambda}(t)\right) T^{\prime}(t)\right] d t>0 .\end{cases}
$$

It turns out that (11) completely characterizes the optimal solutions to (8)).

Theorem 3.1 A function $\widetilde{G}_{\lambda}(\cdot)$ is an optimal solution to (8) if and only if $\widetilde{G}_{\lambda}(\cdot) \in \mathbb{G}$ and $\widetilde{G}_{\lambda}(\cdot)$ satisfies (11).

Proof: We only need to prove the "if" part. For any feasible $G(\cdot)$ in $\mathbb{G}$, we have

$$
\begin{aligned}
& U_{\Delta}\left(\lambda, \widetilde{G}_{\lambda}(\cdot)\right)-U_{\Delta}(\lambda, G(\cdot)) \\
= & \int_{0}^{1}\left[u\left(W_{\Delta}-\widetilde{G}_{\lambda}(z)\right)-u\left(W_{\Delta}-G(z)\right)\right] T^{\prime}(z) d z+\int_{0}^{1} \lambda\left(\widetilde{G}_{\lambda}(z)-G(z)\right) d z \\
\geqslant & \int_{0}^{1} u^{\prime}\left(W_{\Delta}-\widetilde{G}_{\lambda}(z)\right)\left(G(z)-\widetilde{G}_{\lambda}(z)\right) T^{\prime}(z) d z-\int_{0}^{1} \lambda\left(G(z)-\widetilde{G}_{\lambda}(z)\right) d z \\
= & \int_{0}^{1} N_{\lambda}^{\prime}(z)\left(G(z)-\widetilde{G}_{\lambda}(z)\right) d z=\int_{0}^{1} N_{\lambda}(t)\left(\widetilde{G}_{\lambda}^{\prime}(t)-G^{\prime}(t)\right) d t \geqslant 0 .
\end{aligned}
$$

Hence, $\widetilde{G}_{\lambda}(\cdot)$ is optimal for (88) .

The above theorem establishes a general characterization result for the optimal solutions of (8)). This result, however, is only implicit as an optimal $\widetilde{G}_{\lambda}(\cdot)$ appears on both sides of (11). Moreover, the derivative of $\widetilde{G}_{\lambda}(z)$ is undetermined when $N_{\lambda}(z)=0$. In the next two sections, we will apply this general result to derive the solutions.

\section{Model with Yaari's Dual Criterion}

When $u(x) \equiv x$, the corresponding $V^{r d u}$ reduces to the so-called Yaari's dual criterion (Yaari 1987). In this section we solve our insurance problem with Yaari's criterion by applying Theorem 3.1 . In this case, the condition (11) is greatly simplified. Indeed, when $u(x) \equiv x$, (11) reduces to 


$$
\widetilde{G}_{\lambda}^{\prime}(z) \stackrel{\text { a.e. }}{=} \begin{cases}0, & \text { if } \int_{z}^{1}\left(\lambda-T^{\prime}(t)\right) d t=\lambda(1-z)-(1-T(z))<0, \\ \in\left[0,\left(F_{X}^{-1}\right)^{\prime}(z)\right], & \text { if } \int_{z}^{1}\left(\lambda-T^{\prime}(t)\right) d t=\lambda(1-z)-(1-T(z))=0, \\ \left(F_{X}^{-1}\right)^{\prime}(z), & \text { if } \int_{z}^{1}\left(\lambda-T^{\prime}(t)\right) d t=\lambda(1-z)-(1-T(z))>0 .\end{cases}
$$

It should be noted that although $u(x) \equiv x$ is not strictly concave here, the uniqueness of optimal solution to (8) is implied by the characterizing condition (12).

To apply (12), we need to compare $\lambda$ and $\frac{1-T(z)}{1-z}$. Define $f(z):=\frac{1-T(z)}{1-z}, z \in[0,1)$.

Lemma 4.1 The function $f(\cdot)$ is a continuous function on $[0,1)$. Moreover, under Assumption 2.3, there exists a unique $a \in(0, b)$ such that $f(\cdot)$ is strictly decreasing on $[0, a]$ and strictly increasing on $[a, 1)$.

ProOF: We have $f^{\prime}(z)=\frac{(1-T(z))-T^{\prime}(z)(1-z)}{(1-z)^{2}}, z \in[0,1)$. Let $p(z):=(1-T(z))-T^{\prime}(z)(1-z)$. Then $p^{\prime}(z)=-T^{\prime}(z)+T^{\prime}(z)-T^{\prime \prime}(z)(1-z)=-T^{\prime \prime}(z)(1-z)$. It follows from Assumption 2.3 that $p^{\prime}(z)>0$ for $z \in(0, b)$ and $p^{\prime}(z)<0$ for $z \in(b, 1)$. Moreover, $p(0+)=1-T^{\prime}(0+)<0, p(b)=$ $(1-T(b))-T^{\prime}(b)(1-b)=\left(\frac{1-T(b)}{1-b}-T^{\prime}(b)\right)(1-b)>0$, and $p(1-)=\lim _{z \uparrow 1}\left(\frac{1-T(z)}{1-z}-T^{\prime}(z)\right)(1-z) \geqslant 0$ (noting $T(\cdot)$ is strictly convex on $[b, 1]$ ). So, there exists $a \in(0, b)$ such that $p(z)<0$ for $z \in[0, a)$ and $p(z)>0$ for $z \in(a, 1)$. The desired result follows.

Clearly, $f(0)=1, f(1-)=+\infty$. Set $\widehat{\lambda}:=f(a)<f(0)=1$. From the proof of Lemma 4.1, $a$ is determined by $T^{\prime}(a)=\frac{1-T(a)}{1-a}$. Let $c \in(a, 1]$ be the unique scalar such that $f(c)=1$ or $T(c)=c$. See Figure 1 for the locations of the points $a$ and $c$.

Now, we proceed by considering three cases based on the value of $\lambda$.

Case $4.1 \lambda \leqslant \widehat{\lambda}$.

In this case, $N_{\lambda}(z)=(1-z)(\lambda-f(z))<0 \quad \forall z \in[0, a) \cup(a, 1]$. It then follows from (12) that $\widetilde{G}_{\lambda}^{\prime}(z) \stackrel{\text { a.e. }}{=} 0$; hence $\widetilde{G}_{\lambda}(z)=0 \forall z \in[0,1]$. Thus the corresponding retention $\widetilde{R}_{\lambda}(z)=0 \forall z \in[0, M]$ and indemnity $\widetilde{I}_{\lambda}(z)=z \forall z \in[0, M]$, namely, the optimal contract is a full insurance contract.

Case $4.2 \widehat{\lambda}<\lambda<1$. 
By Lemma 4.1, there exist unique $x_{0} \in(0, a)$ and $y_{0} \in(a, c)$ such that $f\left(x_{0}\right)=f\left(y_{0}\right)=\lambda$. Accordingly, we have

$$
N_{\lambda}(z)= \begin{cases}<0, & \text { if } 0<z<x_{0} \\ >0, & \text { if } x_{0}<z<y_{0} \\ <0, & \text { if } y_{0}<z<1\end{cases}
$$

Hence, (12) leads to the following function:

$$
\widetilde{G}_{\lambda}(z)= \begin{cases}0, & \text { if } 0 \leqslant z<x_{0} \\ F_{X}^{-1}(z)-F_{X}^{-1}\left(x_{0}\right), & \text { if } x_{0} \leqslant z<y_{0} \\ F_{X}^{-1}\left(y_{0}\right)-F_{X}^{-1}\left(x_{0}\right), & \text { if } y_{0} \leqslant z \leqslant 1\end{cases}
$$

The corresponding retention and indemnity functions are, respectively,

$$
\widetilde{R}_{\lambda}(z) \equiv \widetilde{G}_{\lambda}\left(F_{X}(z)\right)= \begin{cases}0, & \text { if } 0 \leqslant z<F_{X}^{-1}\left(x_{0}\right), \\ z-F_{X}^{-1}\left(x_{0}\right), & \text { if } F_{X}^{-1}\left(x_{0}\right) \leqslant z<F_{X}^{-1}\left(y_{0}\right), \\ F_{X}^{-1}\left(y_{0}\right)-F_{X}^{-1}\left(x_{0}\right), & \text { if } F_{X}^{-1}\left(y_{0}\right) \leqslant z \leqslant M,\end{cases}
$$

and

$$
\widetilde{I}_{\lambda}(z) \equiv z-\widetilde{R}_{\lambda}(z)= \begin{cases}z, & \text { if } 0 \leqslant z<F_{X}^{-1}\left(x_{0}\right), \\ F_{X}^{-1}\left(x_{0}\right), & \text { if } F_{X}^{-1}\left(x_{0}\right) \leqslant z<F_{X}^{-1}\left(y_{0}\right), \\ z-F_{X}^{-1}\left(y_{0}\right)+F_{X}^{-1}\left(x_{0}\right), & \text { if } F_{X}^{-1}\left(y_{0}\right) \leqslant z \leqslant M .\end{cases}
$$

The corresponding indemnity function is illustrated by Figure 2. Qualitatively, the insurance covers not only large losses (when $z \geqslant F_{X}^{-1}\left(y_{0}\right)$ ) but also small losses (when $z<F_{X}^{-1}\left(x_{0}\right)$ ), and the compensation is a constant for the median range of losses. We term such a contract a threefold one. The need for small loss coverage along with its connection to the probability weighting are amply discussed in Bernard et al. (2015). However, in Bernard et al. (2015) the optimal indemnity is strictly decreasing in some ranges of the losses. Such a contract may incentivize the insured to hide partial losses in order to get more compensations. In contrast, both our indemnity and retention are increasing functions of the loss, which will rule out this sort of moral hazard.

Case $4.31 \leqslant \lambda<+\infty$ 


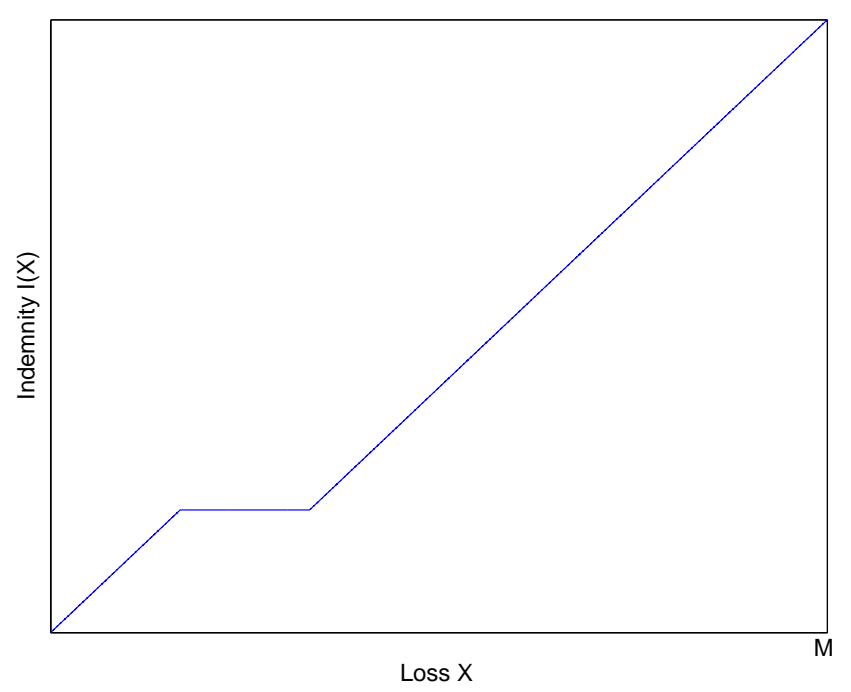

Figure 2: A threefold contract.

By Lemma 4.1, there exists a unique $z_{0} \in[c, 1]$ such that $f\left(z_{0}\right)=\lambda$. Thus

$$
N_{\lambda}(z)= \begin{cases}>0, & \text { if } 0<z<z_{0} \\ <0, & \text { if } z_{0}<z<1 .\end{cases}
$$

By (12), we have

$$
\widetilde{G}_{\lambda}(z)= \begin{cases}F_{X}^{-1}(z), & \text { if } 0 \leqslant z<z_{0} \\ F_{X}^{-1}\left(z_{0}\right), & \text { if } z_{0} \leqslant z \leqslant 1\end{cases}
$$

So

$$
\widetilde{I}_{\lambda}(z) \equiv z-\widetilde{R}_{\lambda}(z)= \begin{cases}0, & \text { if } 0 \leqslant z<F_{X}^{-1}\left(z_{0}\right), \\ z-F_{X}^{-1}\left(z_{0}\right), & \text { if } F_{X}^{-1}\left(z_{0}\right) \leqslant z \leqslant M .\end{cases}
$$

This contract is a standard deductible contract in which only losses above a deductible point will be covered.

We now summarize our results. Define

$$
\bar{G}(z)= \begin{cases}F_{X}^{-1}(z), & \text { if } 0 \leqslant z<c \\ F_{X}^{-1}(c), & \text { if } c \leqslant z \leqslant 1\end{cases}
$$

and let $K_{c}:=\int_{0}^{1} \bar{G}(z) d z$. Clearly $K_{c} \leqslant \int_{0}^{1} F_{X}^{-1}(z) d z=E[X]$. 
Proposition 4.1 Under Yaari's criterion, $u(x) \equiv x$, and Assumptions 2.1 and 2.3, we have the following conclusions:

(i) If $\Delta=0$, then the optimal solution to (6) is $G^{*}(z)=0,0 \leqslant z \leqslant 1$.

(ii) If $0<\Delta<K_{c}$, then the optimal solution to (6) is

$$
G^{*}(z)= \begin{cases}0, & \text { if } 0 \leqslant z<d, \\ F_{X}^{-1}(z)-F_{X}^{-1}(d), & \text { if } d \leqslant z<e, \\ F_{X}^{-1}(e)-F_{X}^{-1}(d), & \text { if } e \leqslant z \leqslant 1,\end{cases}
$$

where $(d, e)$ is the unique pair satisfying $0 \leqslant d<a<e \leqslant c, f(d)=f(e)$ and $\int_{0}^{1} G^{*}(z) d z=\Delta$.

(iii) If $K_{c} \leqslant \Delta \leqslant E[X]$, then the optimal solution to ([6]) is

$$
G^{*}(z)= \begin{cases}F_{X}^{-1}(z), & \text { if } 0 \leqslant z<q, \\ F_{X}^{-1}(q), & \text { if } q \leqslant z \leqslant 1,\end{cases}
$$

where $q$ the unique number satisfying $c \leqslant q$ and $\int_{0}^{1} G^{*}(z) d z=\Delta$.

\section{PROOF:}

(i) When $\Delta=0$, the optimal solution to (6) is trivially $G^{*}(z)=0,0 \leqslant z \leqslant 1$.

(ii) When $0<\Delta<K_{c}$, there exists a unique pair $(d, e)$ such that $0 \leqslant d<a<e \leqslant c, f(d)=f(e)$ and $\int_{0}^{1} G^{*}(z) d z=\Delta$ where $G^{*}$ is defined by (18). The existence of this pair follows from the condition that $\Delta<K_{c}$ and the definition of $K_{c}$, whereas the uniqueness comes from the requirement that $f(d)=f(e)$ and $\int_{0}^{1} G^{*}(z) d z=\Delta$. Letting $\lambda \equiv \lambda_{\Delta}:=f(d)$, it is easy to show that $G^{*}(\cdot)$ satisfies (12) under $\lambda$, corresponding to the aforementioned Case 4.2. This implies that $G^{*}(\cdot)$ is optimal for $(\underline{6})$ under $\Delta$.

(iii) When $K_{c} \leqslant \Delta \leqslant E[X]$, a case corresponding to Case 4.3, the desired result can be derived similarly as in (ii).

The proof is completed.

We are now in the position to state our main result in terms of the premium $\pi$ and the indemnity function $I(\cdot)$. Denote by $\pi_{c}:=(1+\rho)\left(E[X]-K_{c}\right)$. 
Theorem 4.2 Under Yaari's criterion, $u(x) \equiv x$, and Assumptions [2.1 and 2.3, the optimal indemnity function $I^{*}(\cdot)$ to Problem (2) is given as

(i) If $\pi \geqslant(1+\rho) E[X]$, then $I^{*}(z)=z \forall z \in[0, M]$.

(ii) If $\pi_{c}<\pi<(1+\rho) E[X]$, then

$$
I^{*}(z)= \begin{cases}z, & \text { if } 0 \leqslant z<F_{X}^{-1}(d), \\ F_{X}^{-1}(d), & \text { if } F_{X}^{-1}(d) \leqslant z<F_{X}^{-1}(e), \\ z-F_{X}^{-1}(e)+F_{X}^{-1}(d), & \text { if } F_{X}^{-1}(e) \leqslant z \leqslant M,\end{cases}
$$

where $(d, e)$ is the unique pair satisfying $0 \leqslant d<a<e \leqslant c, f(d)=f(e)$ and $E\left[I^{*}(X)\right]=\frac{\pi}{1+\rho}$.

(iii) If $0 \leqslant \pi \leqslant \pi_{c}$, then

$$
I^{*}(z)= \begin{cases}0, & \text { if } 0 \leqslant z<F_{X}^{-1}(q), \\ z-F_{X}^{-1}(q), & \text { if } F_{X}^{-1}(q) \leqslant z \leqslant M,\end{cases}
$$

where $q$ is the unique scalar satisfying $c \leqslant q$ and $E\left[I^{*}(X)\right]=\frac{\pi}{1+\rho}$.

ProOf: Since $\Delta=E[X]-\frac{\pi}{1+\rho}$, the constraint $E[R(X)] \equiv \int_{0}^{1} G_{R(X)}(z) d z=\Delta$ is equivalent to that $E[I(X)]=E[X]-E[R(X)]=\frac{\pi}{1+\rho}$. The desired result is then a direct consequence of Proposition 4.1.

The economic interpretation of this result is clear. When the premium is small $\left(0 \leqslant \pi \leqslant \pi_{c}\right)$, the insurance only compensates large losses in excess of certain amount. When the premium is in middle range $\left(\pi_{c}<\pi<(1+\rho) E[X]\right)$, the contract is a threefold one, covering both small and large losses. When the premium is sufficiently large $(\pi \geqslant(1+\rho) E[X])$, it is a full coverage.

It is interesting to investigate the comparative statics of the point $\pi_{c}$ (in terms of $c$ ) that triggers the coverage for small losses. In fact, as $K_{c}=\int_{0}^{c} F_{X}^{-1}(z) d z+F_{X}^{-1}(c)(1-c)$, we have $\frac{\partial K_{c}}{\partial c}=(1-$ $c)\left(F_{X}^{-1}\right)^{\prime}(c)$. However, $\pi_{c}=(1+\rho)\left(E[X]-K_{c}\right)$; hence $\frac{\partial \pi_{c}}{\partial c}=(1+\rho)(c-1)\left(F_{X}^{-1}\right)^{\prime}(c)<0$. This implies that the insurer is more willing to be protected against small losses if his weighting function has a bigger $c$. This is consistent with the fact that a bigger $c$ renders a larger concave domain of the probability weighting that overweighs small losses (refer to Figure 1). 


\section{Model with the RDU Criterion}

In this section we study the general RDU model in which the utility function is strictly concave. Compared with the Yaari model, solving the corresponding insurance problem calls for a more delicate analysis.

For any twice differentiable function $f$ with $f^{\prime}(x) \neq 0$, define its Arrow-Pratt measure of absolute risk aversion $A_{f}(x):=-\frac{f^{\prime \prime}(x)}{f^{\prime}(x)}$. We now introduce the following assumptions.

Assumption 5.1 (Strictly Concave Utility) The utility function $u: \mathbb{R}^{+} \mapsto \mathbb{R}^{+}$is strictly increasing and twice differentiable. Furthermore, $u^{\prime}$ is strictly decreasing.

Assumption $\mathbf{5 . 2}$ (i) The function $A_{u}(z)$ is decreasing on $(0, \infty)$.

(ii) $A_{T}(z)>A_{u}\left(W-F_{X}^{-1}(z)\right)\left(F_{X}^{-1}\right)^{\prime}(z) \forall z \in(0, a]$.

Assumption 5.1 is to replace Assumption [2.2, ensuring a genuine RDU criterion. Assumption 5.2 (i) requires that the absolute risk aversion measure of the utility function $u$ be decreasing, which holds true for many frequently used utility functions including logarithmic, power and exponential utilities. In general, experimental and empirical evidences are consistent with the decreasing absolute risk aversion; see e.g. Friend, Irwin and Blume, Marshall (1975). On the other hand, $A_{T}(z)$, $z \in(0, a]$, measures the level of probability weighting for small losses. The economical interpretation of Assumption 5.2.(ii) is, therefore, that the degree of the insured's concern for small losses is sufficiently large relative to the absolute risk aversion of the utility function. Note that Assumption 5.2-(ii) is automatically satisfied when $F_{X}^{-1}(z)=0, \forall z \in[0, a]$, which is equivalent to $\mathbb{P}(X=0) \geqslant a$. In practice, $\mathbb{P}(X=0) \geqslant 0.5$ is a plausible assumption for many insurance products such as automobile and house insurance. On the other hand, $a$ is very small for many commonly used inverse- $S$ shaped weighting functions. Take Tversky and Kahneman's weighting function (3) as an example, $a \approx 0.013$ when $\theta=0.3, a \approx 0.07$ when $\theta=0.5$, and $a \approx 0.166$ when $\theta=0.8$. In these cases, Assumption 5.2 (ii) holds automatically.

Problem (66) has trivial solutions in the following two cases. When $\Delta=0$, the optimal solution is $G^{*}(z)=0 \forall z \in[0,1]$, corresponding to a full coverage. When $\Delta=E[X]$, the optimal solution is $G^{*}(z)=F_{X}^{-1}(z) \forall z \in[0,1]$ as it is the only feasible solution, corresponding to no coverage.

So we are interested in only the case $0<\Delta<E[X]$. It follows from Proposition C.1 in Appendix

C that there exists $\lambda^{*}$ such that $\widetilde{G}_{\lambda^{*}}(\cdot)$ is optimal solution to (8) under $\lambda^{*}$ and $\int_{0}^{1} \widetilde{G}_{\lambda^{*}}(z) d z=\Delta$. 
Furthermore, recall that we have proved that (8) has a unique solution when $u$ is strictly concave and (11) provides the necessary and sufficient condition for the optimal solution.

Lemma 5.1 For any $G(\cdot) \in \mathbb{G}$, if there exists $z \in(0,1)$ such that $\lambda-u^{\prime}\left(W_{\Delta}-G(z)\right) T^{\prime}(z)=$ $\int_{z}^{1}\left[\lambda-u^{\prime}\left(W_{\Delta}-G(t)\right) T^{\prime}(t)\right] d t=0$, then $z \leqslant a$.

ProOf: From $\lambda-u^{\prime}\left(W_{\Delta}-G(z)\right) T^{\prime}(z)=0$, it follows $u^{\prime}\left(W_{\Delta}-G(z)\right)=\frac{\lambda}{T^{\prime}(z)}$. Hence, if $z>a$, then

$$
\begin{aligned}
0 & =\int_{z}^{1}\left[\lambda-u^{\prime}\left(W_{\Delta}-G(t)\right) T^{\prime}(t)\right] d t \\
& \leqslant \int_{z}^{1}\left[\lambda-u^{\prime}\left(W_{\Delta}-G(z)\right) T^{\prime}(t)\right] d t=\frac{\lambda}{T^{\prime}(z)}(1-z)\left[T^{\prime}(z)-\frac{1-T(z)}{1-z}\right]<0,
\end{aligned}
$$

where the last inequality is due to Lemma A.1 (i) in Appendix A, noting $z>a$. This is a contradiction.

Lemma 5.2 Under Assumption 5.2, for any $G(\cdot) \in \mathbb{G}, u^{\prime}\left(W_{\Delta}-G(z)\right) T^{\prime}(z)$ is a strictly decreasing function of $z$ on $[0, a]$.

Proof: Noting $W_{\Delta} \geqslant W$, it follows from Assumption 5.2 that $A_{T}(z)>A_{u}\left(W-F_{X}^{-1}(z)\right)\left(F_{X}^{-1}\right)^{\prime}(z) \geqslant$ $A_{u}\left(W_{\Delta}-F_{X}^{-1}(z)\right)\left(F_{X}^{-1}\right)^{\prime}(z) \forall z \in(0, a]$. Now, we compute the partial derivative of $u^{\prime}\left(W_{\Delta}-G(z)\right) T^{\prime}(z)$ with respect to $z \in[0, a]$ :

$$
\begin{aligned}
& \frac{\partial}{\partial z}\left(u^{\prime}\left(W_{\Delta}-G(z)\right) T^{\prime}(z)\right) \\
= & -u^{\prime \prime}\left(W_{\Delta}-G(z)\right) G^{\prime}(z) T^{\prime}(z)+u^{\prime}\left(W_{\Delta}-G(z)\right) T^{\prime \prime}(z) \\
= & u^{\prime}\left(W_{\Delta}-G(z)\right) T^{\prime}(z)\left[A_{u}\left(W_{\Delta}-G(z)\right) G^{\prime}(z)-A_{T}(z)\right] \\
< & u^{\prime}\left(W_{\Delta}-G(z)\right) T^{\prime}(z)\left[A_{u}\left(W_{\Delta}-G(z)\right) G^{\prime}(z)-A_{u}\left(W_{\Delta}-F_{X}^{-1}(z)\right)\left(F_{X}^{-1}\right)^{\prime}(z)\right] \\
\leqslant & u^{\prime}\left(W_{\Delta}-G(z)\right) T^{\prime}(z)\left[A_{u}\left(W_{\Delta}-G(z)\right)\left(F_{X}^{-1}\right)^{\prime}(z)-A_{u}\left(W_{\Delta}-F_{X}^{-1}(z)\right)\left(F_{X}^{-1}\right)^{\prime}(z)\right] \\
\leqslant & u^{\prime}\left(W_{\Delta}-G(z)\right) T^{\prime}(z)\left[A_{u}\left(W_{\Delta}-F_{X}^{-1}(z)\right)\left(F_{X}^{-1}\right)^{\prime}(z)-A_{u}\left(W_{\Delta}-F_{X}^{-1}(z)\right)\left(F_{X}^{-1}\right)^{\prime}(z)\right] \\
= & 0 .
\end{aligned}
$$

The proof is complete.

Now, for any $\lambda \leqslant \widehat{\lambda} u^{\prime}\left(W_{\Delta}\right)$, we have

$$
\int_{z}^{1}\left[\lambda-u^{\prime}\left(W_{\Delta}-\widetilde{G}_{\lambda}(t)\right) T^{\prime}(t)\right] d t \leqslant \int_{z}^{1}\left[\widehat{\lambda} u^{\prime}\left(W_{\Delta}\right)-u^{\prime}\left(W_{\Delta}\right) T^{\prime}(t)\right] d t
$$




$$
=u^{\prime}\left(W_{\Delta}\right) \int_{z}^{1}\left[\widehat{\lambda}-T^{\prime}(t)\right] d t=u^{\prime}\left(W_{\Delta}\right)(1-z)\left[\widehat{\lambda}-\frac{1-T(z)}{1-z}\right]<0,
$$

where the last inequality is due to Lemma 4.1. Hence $\widetilde{G}_{\lambda}(z)=0 \forall z \in[0,1]$ is the only solution satisfying (11). However, $\int_{0}^{1} \widetilde{G}_{\lambda}(z) d z=0<\Delta$, a contradiction. Therefore, only when $\lambda>\widehat{\lambda} u^{\prime}\left(W_{\Delta}\right)$ is it possible for (11) to hold.

Fixing $\lambda>\widehat{\lambda} u^{\prime}\left(W_{\Delta}\right)$, we now analyze the shape of the function $\widetilde{G}_{\lambda}(\cdot)$ that satisfies (11). Assume that $\widetilde{G}_{\lambda}(1)=k<W_{\Delta}$. We have $N_{\lambda}(1)=0$ and $\lambda-u^{\prime}\left(W_{\Delta}-k\right) T^{\prime}(1-)<0$ since $T^{\prime}(1-)=+\infty$. So, $\widetilde{G}_{\lambda}^{\prime}(z)=0$ when $z$ is close to 1 since $N_{\lambda}(z)<0$ for such $z$. Hence, $\widetilde{G}_{\lambda}(z) \equiv k \forall z \in\left[z_{1}, 1\right]$ for some $z_{1} \in[0,1)$, at which $N_{\lambda}\left(z_{1}\right)=0$ and $N_{\lambda}(z)<0$ for $\forall z \in\left(z_{1}, 1\right)$. Next, we consider three cases respectively depending on the value of $k$.

(A) If $k>W_{\Delta}-\left(u^{\prime}\right)^{-1}\left(\frac{\lambda}{\lambda}\right)\left(\right.$ i.e. $\lambda<\widehat{\lambda} u^{\prime}\left(W_{\Delta}-k\right)$ ), then we have, $\forall z \in[0,1)$

$$
\begin{aligned}
\int_{z}^{1}\left[\lambda-u^{\prime}\left(W_{\Delta}-k\right) T^{\prime}(t)\right] d t & <\int_{z}^{1}\left[\widehat{\lambda} u^{\prime}\left(W_{\Delta}-k\right)-u^{\prime}\left(W_{\Delta}-k\right) T^{\prime}(t)\right] d t \\
& =u^{\prime}\left(W_{\Delta}-k\right)(1-z)\left[\widehat{\lambda}-\frac{1-T(z)}{1-z}\right] \leqslant 0 .
\end{aligned}
$$

It then follows from (11) that $\widetilde{G}_{\lambda}(z) \equiv k=\widetilde{G}_{\lambda}(0)=0$. However, $0=k>W_{\Delta}-\left(u^{\prime}\right)^{-1}\left(\frac{\lambda}{\lambda}\right)$, or $\lambda \leqslant \widehat{\lambda} u^{\prime}\left(W_{\Delta}\right)$, leading to a contradiction. So, this case in fact will not take place.

(B) If $k=W_{\Delta}-\left(u^{\prime}\right)^{-1}\left(\frac{\lambda}{\lambda}\right)$, then $z_{1}$ should be $a$. This is because $\int_{a}^{1}\left[\lambda-u^{\prime}\left(W_{\Delta}-k\right) T^{\prime}(t)\right] d t=0$ and $\int_{z}^{1}\left[\lambda-u^{\prime}\left(W_{\Delta}-k\right) T^{\prime}(t)\right] d t=\frac{\lambda}{\lambda}(1-z)\left(\widehat{\lambda}-\frac{1-T(z)}{1-z}\right)<0$ for $z \in(a, 1)$ by Lemma 4.1. Moreover, $\lambda-u^{\prime}\left(W_{\Delta}-k\right) T^{\prime}(a)=0$. By Lemma 5.2, $\lambda-u^{\prime}\left(W_{\Delta}-\widetilde{G}_{\lambda}(z)\right) T^{\prime}(z)$ strictly increases with respect to $z \in[0, a]$. It follows that $\lambda-u^{\prime}\left(W_{\Delta}-\widetilde{G}_{\lambda}(z)\right) T^{\prime}(z)<0$ for $z \in[0, a)$. Then (11) implies $\widetilde{G}_{\lambda}^{\prime}(z)=0$ for $z \in(0, a)$. As a result, $k=\widetilde{G}_{\lambda}(a)=\widetilde{G}_{\lambda}(0)=0$, or $\lambda=\widehat{\lambda} u^{\prime}\left(W_{\Delta}\right)$, which is a contradiction. So, again, this case will not occur.

(C) If $k<W_{\Delta}-\left(u^{\prime}\right)^{-1}\left(\frac{\lambda}{\lambda}\right)$, then $z_{1} \in(a, 1)$ exists. By Lemma [5.1, we have $\lambda-u^{\prime}\left(W_{\Delta}-k\right) T^{\prime}\left(z_{1}\right)>$ 0 . Hence, there may or may not exist $z_{2} \in(0,1)$ such that $N_{\lambda}\left(z_{2}\right)=0$ and $N_{\lambda}(z)>0$ for $z \in\left(z_{2}, z_{1}\right)$. We now discuss four subcases depending on the existence and location of $z_{2}$.

(C.1) If $z_{2}$ does not exist or $z_{2}=0$ (i.e. $N_{\lambda}(z)>0$ for $\left.z \in\left(0, z_{1}\right)\right)$, then by (11), $\widetilde{G}_{\lambda}^{\prime}(z)=\left(F_{X}^{-1}\right)^{\prime}(z)$ for $z \in\left(0, z_{1}\right)$. Combined with the fact that $\widetilde{G}_{\lambda}(0)=0$, we have:

$$
\widetilde{G}_{\lambda}(z)= \begin{cases}F_{X}^{-1}(z), & \text { if } 0 \leqslant z<z_{1}, \\ F_{X}^{-1}\left(z_{1}\right), & \text { if } z_{1} \leqslant z \leqslant 1 .\end{cases}
$$

This corresponds to a deductible contract. 
(C.2) If $z_{2}$ exists and $z_{2} \in(0, a]$, then $\widetilde{G}_{\lambda}^{\prime}(z)=\left(F_{X}^{-1}\right)^{\prime}(z)$ for $z \in\left(z_{2}, z_{1}\right)$ in view of (11). Combining the property of $z_{1}$ and $z_{2}$, we deduce $\lambda-u^{\prime}\left(W_{\Delta}-\widetilde{G}_{\lambda}\left(z_{2}\right)\right) T^{\prime}\left(z_{2}\right) \leqslant 0$. Then, using Lemma 5.2, we have $\lambda-u^{\prime}\left(W_{\Delta}-\widetilde{G}_{\lambda}(z)\right) T^{\prime}(z)<0$ for $z \in\left[0, z_{2}\right)$. It follows from (11) that $\widetilde{G}_{\lambda}^{\prime}(z)=0$ for $z \in\left(0, z_{2}\right)$. In this case, we can express $\widetilde{G}_{\lambda}(\cdot)$ as follows

$$
\widetilde{G}_{\lambda}(z)= \begin{cases}0, & \text { if } 0 \leqslant z<z_{2}, \\ F_{X}^{-1}(z)-F_{X}^{-1}\left(z_{2}\right), & \text { if } z_{2} \leqslant z<z_{1}, \\ F_{X}^{-1}\left(z_{1}\right)-F_{X}^{-1}\left(z_{2}\right), & \text { if } z_{1} \leqslant z \leqslant 1 .\end{cases}
$$

This is the threefold contract, depicted in Figure 2.

(C.3) If $z_{2}$ exists and $z_{2} \in(b, 1)$ (recall that $b$ is the turning point where the weighting function $T(\cdot)$ changes from being concave to convex), then a similar analysis as in Case C.2 shows that $\lambda-$ $u^{\prime}\left(W_{\Delta}-\widetilde{G}_{\lambda}\left(z_{1}\right)\right) T^{\prime}\left(z_{1}\right)>0$ and $\lambda-u^{\prime}\left(W_{\Delta}-\widetilde{G}_{\lambda}\left(z_{2}\right)\right) T^{\prime}\left(z_{2}\right)<0$. This means $u^{\prime}\left(W_{\Delta}-\widetilde{G}_{\lambda}\left(z_{2}\right)\right) T^{\prime}\left(z_{2}\right)>$ $u^{\prime}\left(W_{\Delta}-\widetilde{G}_{\lambda}\left(z_{1}\right)\right) T^{\prime}\left(z_{1}\right)$. However, $u^{\prime}\left(W_{\Delta}-\widetilde{G}_{\lambda}\left(z_{1}\right)\right) \geqslant u^{\prime}\left(W_{\Delta}-\widetilde{G}_{\lambda}\left(z_{2}\right)\right)>0$ and $T^{\prime}\left(z_{1}\right)>T^{\prime}\left(z_{2}\right)>0$, which is a contradiction. So, this case is not feasible.

(C.4) If $z_{2}$ exists and $z_{2} \in(a, b]$, then $\lambda-u^{\prime}\left(W_{\Delta}-\widetilde{G}_{\lambda}\left(z_{2}\right)\right) T^{\prime}\left(z_{2}\right)<0$. We prove $\widetilde{G}_{\lambda}(z) \equiv$ $\widetilde{G}_{\lambda}\left(z_{2}\right) \forall z \in\left[0, z_{2}\right]$. In fact, if it is false, then there exists $z_{3}$ such that $\int_{z_{3}}^{z_{2}}\left[\lambda-u^{\prime}\left(W_{\Delta}-\widetilde{G}_{\lambda}\left(z_{2}\right)\right) T^{\prime}(t)\right] d t=$ 0 and $\int_{z}^{z_{2}}\left[\lambda-u^{\prime}\left(W_{\Delta}-\widetilde{G}_{\lambda}\left(z_{2}\right)\right) T^{\prime}(t)\right] d t<0$ for $z \in\left(z_{3}, z_{2}\right)$. However, $\lambda-u^{\prime}\left(W_{\Delta}-\widetilde{G}_{\lambda}\left(z_{2}\right)\right) T^{\prime}(z)<\lambda-$ $u^{\prime}\left(W_{\Delta}-\widetilde{G}_{\lambda}\left(z_{2}\right)\right) T^{\prime}\left(z_{2}\right)<0$ for $z \in\left(z_{3}, z_{2}\right)$ since $z_{2} \in(a, b]$. So, $\int_{z_{3}}^{z_{2}}\left[\lambda-u^{\prime}\left(W_{\Delta}-\widetilde{G}_{\lambda}\left(z_{2}\right)\right) T^{\prime}(t)\right] d t<(\lambda-$ $\left.u^{\prime}\left(W_{\Delta}-\widetilde{G}_{\lambda}\left(z_{2}\right)\right) T^{\prime}\left(z_{2}\right)\right)\left(z_{2}-z_{3}\right)<0$, arriving at a contradiction. Therefore, $k=F_{X}^{-1}\left(z_{1}\right)-F_{X}^{-1}\left(z_{2}\right)$. From $\int_{z_{1}}^{1}\left[\lambda-u^{\prime}\left(W_{\Delta}-k\right) T^{\prime}(t)\right] d t=0$, it follows $\lambda=u^{\prime}\left(W_{\Delta}-k\right) \frac{1-T\left(z_{1}\right)}{1-z_{1}}=u^{\prime}\left(W_{\Delta}+F_{X}^{-1}\left(z_{2}\right)-\right.$ $\left.F_{X}^{-1}\left(z_{1}\right)\right) \frac{1-T\left(z_{1}\right)}{1-z_{1}}$. However,

$$
\begin{aligned}
& \int_{z_{2}}^{z_{1}}\left[u^{\prime}\left(W_{\Delta}+F_{X}^{-1}\left(z_{2}\right)-F_{X}^{-1}\left(z_{1}\right)\right) \frac{1-T\left(z_{1}\right)}{1-z_{1}}-u^{\prime}\left(W_{\Delta}+F_{X}^{-1}\left(z_{2}\right)-F_{X}^{-1}(t)\right) T^{\prime}(t)\right] d t \\
> & \int_{z_{2}}^{z_{1}}\left[u^{\prime}\left(W_{\Delta}+F_{X}^{-1}\left(z_{2}\right)-F_{X}^{-1}\left(z_{1}\right)\right) \frac{1-T\left(z_{1}\right)}{1-z_{1}}-u^{\prime}\left(W_{\Delta}+F_{X}^{-1}\left(z_{2}\right)-F_{X}^{-1}\left(z_{1}\right)\right) T^{\prime}(t)\right] d t \\
= & u^{\prime}\left(W_{\Delta}+F_{X}^{-1}\left(z_{2}\right)-F_{X}^{-1}\left(z_{1}\right)\right)\left(z_{1}-z_{2}\right)\left[\frac{1-T\left(z_{1}\right)}{1-z_{1}}-\frac{T\left(z_{1}\right)-T\left(z_{2}\right)}{z_{1}-z_{2}}\right]>0,
\end{aligned}
$$

where the last inequality follows from Lemma A.1-(ii) in Appendix A. This is a contradiction. So, the current case will not occur either.

To summarize, for any $\lambda>\widehat{\lambda} u^{\prime}\left(W_{\Delta}\right)$, only deductible and threefold contracts are possibly optimal, stipulated in Case C.1 and Case C.2 above. Next, we investigate these two cases more closely.

Define a function $h_{\Delta}(\cdot)$ on $[a, c]$ as follows:

$$
h_{\Delta}(z):=\int_{0}^{z}\left[\frac{u^{\prime}\left(W_{\Delta}-F_{X}^{-1}(z)\right)(1-T(z))}{1-z}-u^{\prime}\left(W_{\Delta}-F_{X}^{-1}(t)\right) T^{\prime}(t)\right] d t .
$$


Then, by using Lemma 5.2 and the fact that $\frac{1-T(a)}{1-a}=T^{\prime}(a)$, we have

$$
h_{\Delta}(a)=\int_{0}^{a}\left[u^{\prime}\left(W_{\Delta}-F_{X}^{-1}(a)\right) T^{\prime}(a)-u^{\prime}\left(W_{\Delta}-F_{X}^{-1}(t)\right) T^{\prime}(t)\right] d t<0 .
$$

Recalling that $T(c)=c$, we have

$$
\begin{aligned}
h_{\Delta}(c) & =\int_{0}^{c}\left[\frac{u^{\prime}\left(W_{\Delta}-F_{X}^{-1}(c)\right)(1-T(c))}{1-c}-u^{\prime}\left(W_{\Delta}-F_{X}^{-1}(t)\right) T^{\prime}(t)\right] d t \\
& =\int_{0}^{c}\left[u^{\prime}\left(W_{\Delta}-F_{X}^{-1}(c)\right)-u^{\prime}\left(W_{\Delta}-F_{X}^{-1}(t)\right) T^{\prime}(t)\right] d t \\
& >u^{\prime}\left(W_{\Delta}-F_{X}^{-1}(c)\right) c-\int_{0}^{c}\left[u^{\prime}\left(W_{\Delta}-F_{X}^{-1}(c)\right) T^{\prime}(t)\right] d t=0 .
\end{aligned}
$$

Moreover, we take the derivative of $h_{\Delta}(z)$ with respect to $z \in[a, c]$ to obtain

$$
\begin{aligned}
h_{\Delta}^{\prime}(z)= & -u^{\prime}\left(W_{\Delta}-F_{X}^{-1}(z)\right) T^{\prime}(z)+u^{\prime}\left(W_{\Delta}-F_{X}^{-1}(z)\right) \frac{1-T(z)}{1-z} \\
& -u^{\prime \prime}\left(W_{\Delta}-F_{X}^{-1}(z)\right) \frac{1-T(z)}{1-z} z\left(F_{X}^{-1}\right)^{\prime}(z)+u^{\prime}\left(W_{\Delta}-F_{X}^{-1}(z)\right) z \frac{\frac{1-T(z)}{1-z}-T^{\prime}(z)}{1-z} \\
= & u^{\prime}\left(W_{\Delta}-F_{X}^{-1}(z)\right)\left(\frac{1-T(z)}{1-z}-T^{\prime}(z)\right)-u^{\prime \prime}\left(W_{\Delta}-F_{X}^{-1}(z)\right) \frac{1-T(z)}{1-z} z\left(F_{X}^{-1}\right)^{\prime}(z) \\
& +u^{\prime}\left(W_{\Delta}-F_{X}^{-1}(z)\right) z \frac{\frac{1-T(z)}{1-z}-T^{\prime}(z)}{1-z}>0 .
\end{aligned}
$$

Hence, there exists a unique point $l_{\Delta} \in(a, c)$ such that $h_{\Delta}\left(l_{\Delta}\right)=0, h_{\Delta}(z)<0$ for $z \in\left(a, l_{\Delta}\right)$, and $h_{\Delta}(z)>0$ for $z \in\left(l_{\Delta}, c\right)$.

Define

$$
\underline{G}(z)= \begin{cases}F_{X}^{-1}(z), & \text { if } 0 \leqslant z<l_{\Delta}, \\ F_{X}^{-1}\left(l_{\Delta}\right), & \text { if } l_{\Delta} \leqslant z \leqslant 1,\end{cases}
$$

and $K_{\Delta}:=\int_{0}^{1} \underline{G}(z) d z$

Proposition 5.1 If $K_{\Delta} \leqslant \Delta<E[X]$, then the optimal solution to (6) is

$$
G^{*}(z)= \begin{cases}F_{X}^{-1}(z), & \text { if } 0 \leqslant z<f, \\ F_{X}^{-1}(f), & \text { if } f \leqslant z \leqslant 1,\end{cases}
$$

where $f$ is the unique scalar such that $f \geqslant l_{\Delta}$ and $\int_{0}^{1} G^{*}(z) d z=\Delta$.

Proof: The existence of $f$ follows from the monotonicity of $G^{*}$ with respesct to $f$ immediately. Denoting $\lambda_{\Delta}:=u^{\prime}\left(W_{\Delta}-F_{X}^{-1}(f)\right) \frac{1-T(f)}{1-f}$, we need to show that $G^{*}(\cdot)$ satisfies (11) with $\lambda=\lambda_{\Delta}$. 
First, it is straightforward that $\int_{f}^{1}\left[\frac{u^{\prime}\left(W_{\Delta}-F_{X}^{-1}(f)\right)(1-T(f))}{1-f}-u^{\prime}\left(W_{\Delta}-F_{X}^{-1}(f)\right) T^{\prime}(t)\right] d t=0$. Next, we are to prove that $\int_{z}^{f}\left[\frac{u^{\prime}\left(W_{\Delta}-F_{X}^{-1}(f)\right)(1-T(f))}{1-f}-u^{\prime}\left(W_{\Delta}-F_{X}^{-1}(t)\right) T^{\prime}(t)\right] d t>0 \forall z \in(0, f)$. We divide the proof into two cases.

- If $z \in[a, f)$, then

$$
\begin{aligned}
& \int_{z}^{f}\left[\frac{u^{\prime}\left(W_{\Delta}-F_{X}^{-1}(f)\right)(1-T(f))}{1-f}-u^{\prime}\left(W_{\Delta}-F_{X}^{-1}(t)\right) T^{\prime}(t)\right] d t \\
\geqslant & \int_{z}^{f}\left[\frac{u^{\prime}\left(W_{\Delta}-F_{X}^{-1}(f)\right)(1-T(f))}{1-f}-u^{\prime}\left(W_{\Delta}-F_{X}^{-1}(f)\right) T^{\prime}(t)\right] d t \\
= & u^{\prime}\left(W_{\Delta}-F_{X}^{-1}(f)\right)(f-z)\left[\frac{1-T(f)}{1-f}-\frac{1-T(z)}{1-z}\right]>0,
\end{aligned}
$$

where the last inequality is due to Lemma 4.1.

- If $z \in(0, a)$ and $u^{\prime}\left(W_{\Delta}-F_{X}^{-1}(z)\right) T^{\prime}(z) \leqslant \frac{u^{\prime}\left(W_{\Delta}-F_{X}^{-1}(f)\right)(1-T(f))}{1-f}$, then by Lemma 5.2 and the result above, we have

$$
\begin{aligned}
& \int_{z}^{f}\left[\frac{u^{\prime}\left(W_{\Delta}-F_{X}^{-1}(f)\right)(1-T(f))}{1-f}-u^{\prime}\left(W_{\Delta}-F_{X}^{-1}(t)\right) T^{\prime}(t)\right] d t \\
= & \int_{z}^{a}\left[\frac{u^{\prime}\left(W_{\Delta}-F_{X}^{-1}(f)\right)(1-T(f))}{1-f}-u^{\prime}\left(W_{\Delta}-F_{X}^{-1}(t)\right) T^{\prime}(t)\right] d t \\
& +\int_{a}^{f}\left[\frac{u^{\prime}\left(W_{\Delta}-F_{X}^{-1}(f)\right)(1-T(f))}{1-f}-u^{\prime}\left(W_{\Delta}-F_{X}^{-1}(t)\right) T^{\prime}(t)\right] d t \\
> & \int_{z}^{a}\left[\frac{u^{\prime}\left(W_{\Delta}-F_{X}^{-1}(f)\right)(1-T(f))}{1-f}-u^{\prime}\left(W_{\Delta}-F_{X}^{-1}(t)\right) T^{\prime}(t)\right] d t \\
\geqslant & \int_{z}^{a}\left[u^{\prime}\left(W_{\Delta}-F_{X}^{-1}(z)\right) T^{\prime}(z)-u^{\prime}\left(W_{\Delta}-F_{X}^{-1}(t)\right) T^{\prime}(t)\right] d t>0 .
\end{aligned}
$$

If $z \in(0, a)$ and $u^{\prime}\left(W_{\Delta}-F_{X}^{-1}(z)\right) T^{\prime}(z)>\frac{u^{\prime}\left(W_{\Delta}-F_{X}^{-1}(f)\right)(1-T(f))}{1-f}$ holds, then

$$
\begin{aligned}
& \int_{z}^{f}\left[\frac{u^{\prime}\left(W_{\Delta}-F_{X}^{-1}(f)\right)(1-T(f))}{1-f}-u^{\prime}\left(W_{\Delta}-F_{X}^{-1}(t)\right) T^{\prime}(t)\right] d t \\
= & \int_{l_{\Delta}}^{f}\left[\frac{u^{\prime}\left(W_{\Delta}-F_{X}^{-1}(f)\right)(1-T(f))}{1-f}-u^{\prime}\left(W_{\Delta}-F_{X}^{-1}(t)\right) T^{\prime}(t)\right] d t \\
& +\int_{z}^{l_{\Delta}}\left[\frac{u^{\prime}\left(W_{\Delta}-F_{X}^{-1}(f)\right)(1-T(f))}{1-f}-u^{\prime}\left(W_{\Delta}-F_{X}^{-1}(t)\right) T^{\prime}(t)\right] d t \\
\geqslant & \int_{z}^{l_{\Delta}}\left[\frac{u^{\prime}\left(W_{\Delta}-F_{X}^{-1}(f)\right)(1-T(f))}{1-f}-u^{\prime}\left(W_{\Delta}-F_{X}^{-1}(t)\right) T^{\prime}(t)\right] d t \\
\geqslant & \int_{z}^{l_{\Delta}}\left[\frac{u^{\prime}\left(W_{\Delta}-F_{X}^{-1}\left(l_{\Delta}\right)\right)\left(1-T\left(l_{\Delta}\right)\right)}{1-l_{\Delta}}-u^{\prime}\left(W_{\Delta}-F_{X}^{-1}(t)\right) T^{\prime}(t)\right] d t \\
= & \int_{0}^{l_{\Delta}}\left[\frac{u^{\prime}\left(W_{\Delta}-F_{X}^{-1}\left(l_{\Delta}\right)\right)\left(1-T\left(l_{\Delta}\right)\right)}{1-l_{\Delta}}-u^{\prime}\left(W_{\Delta}-F_{X}^{-1}(t)\right) T^{\prime}(t)\right] d t
\end{aligned}
$$




$$
\begin{aligned}
& -\int_{0}^{z}\left[\frac{u^{\prime}\left(W_{\Delta}-F_{X}^{-1}\left(l_{\Delta}\right)\right)\left(1-T\left(l_{\Delta}\right)\right)}{1-l_{\Delta}}-u^{\prime}\left(W_{\Delta}-F_{X}^{-1}(t)\right) T^{\prime}(t)\right] d t \\
= & -\int_{0}^{z}\left[\frac{u^{\prime}\left(W_{\Delta}-F_{X}^{-1}\left(l_{\Delta}\right)\right)\left(1-T\left(l_{\Delta}\right)\right)}{1-l_{\Delta}}-u^{\prime}\left(W_{\Delta}-F_{X}^{-1}(t)\right) T^{\prime}(t)\right] d t>0,
\end{aligned}
$$

where the last inequality is due to

$$
u^{\prime}\left(W_{\Delta}-F_{X}^{-1}(z)\right) T^{\prime}(z)>\frac{u^{\prime}\left(W_{\Delta}-F_{X}^{-1}(f)\right)(1-T(f))}{1-f} \geqslant \frac{u^{\prime}\left(W_{\Delta}-F_{X}^{-1}\left(l_{\Delta}\right)\right)\left(1-T\left(l_{\Delta}\right)\right)}{1-l_{\Delta}},
$$

as $l_{\Delta} \leqslant f$ and the fact that $u^{\prime}\left(W_{\Delta}-F_{X}^{-1}(z)\right) T^{\prime}(z)$ is strictly decreasing on $[0, a]$.

The claim follows now.

Lemma 5.3 If $0<\Delta<K_{\Delta}$, then the corresponding optimal contract is not a deductible one.

ProOF: There exists $\lambda^{*}$ such that $\widetilde{G}_{\lambda^{*}}(\cdot)$ satisfies (11) under $\lambda^{*}$ and $\int_{0}^{1} \widetilde{G}_{\lambda^{*}}(z) d z=\Delta$ (see Appendix C). If $\widetilde{G}_{\lambda^{*}}(\cdot)$ corresponds to a deductible contract, then there exists $\bar{z}$ ( since $\Delta<K_{\Delta}$, we have $\bar{z}<l_{\Delta}$ ) such that

$$
\widetilde{G}_{\lambda^{*}}(z)= \begin{cases}F_{X}^{-1}(z), & \text { if } 0 \leqslant z<\bar{z}, \\ F_{X}^{-1}(\bar{z}), & \text { if } \bar{z} \leqslant z \leqslant 1 .\end{cases}
$$

Since $\widetilde{G}_{\lambda^{*}}(\cdot)$ satisfies (11), we have $\int_{\bar{z}}^{1}\left[\lambda^{*}-u^{\prime}\left(W_{\Delta}-F_{X}^{-1}(\bar{z})\right) T^{\prime}(t)\right] d t=0$, or $\lambda^{*}=u^{\prime}\left(W_{\Delta}-\right.$ $\left.F_{X}^{-1}(\bar{z})\right) \frac{1-T^{\prime}(\bar{z})}{1-\bar{z}}$.

On the other hand, $M(z):=\int_{z}^{\bar{z}}\left[u^{\prime}\left(W_{\Delta}-F_{X}^{-1}(\bar{z})\right) \frac{1-T^{\prime}(\bar{z})}{1-\bar{z}}-u^{\prime}\left(W_{\Delta}-F_{X}^{-1}(t)\right) T^{\prime}(t)\right] d t \geqslant 0$ for $z \in[0, \bar{z}]$. However, by the definition of $l_{\Delta}, h_{\Delta}(\bar{z}) \equiv M(0)=\int_{0}^{\bar{z}}\left[\frac{u^{\prime}\left(W_{\Delta}-F_{X}^{-1}(\bar{z})\right)(1-T(\bar{z}))}{1-\bar{z}}-u^{\prime}\left(W_{\Delta}-\right.\right.$ $\left.\left.F_{X}^{-1}(t)\right) T^{\prime}(t)\right] d t<0$ as $\bar{z}<l_{\Delta}$. Since $M(\cdot)$ is a continuous function, a contradiction arises.

It follows from Lemma 5.3 that, if $0<\Delta<K_{\Delta}$, the optimal contract (which always exists) can only be a threefold one, corresponding to Case C.2. We are now led to the following proposition.

Proposition 5.2 If $0<\Delta<K_{\Delta}$, then the optimal solution to (16) is given as

$$
G^{*}(z)= \begin{cases}0, & \text { if } 0 \leqslant z<z_{2}, \\ F_{X}^{-1}(z)-F_{X}^{-1}\left(z_{2}\right), & \text { if } z_{2} \leqslant z<z_{1}, \\ F_{X}^{-1}\left(z_{1}\right)-F_{X}^{-1}\left(z_{2}\right), & \text { if } z_{1} \leqslant z \leqslant 1,\end{cases}
$$


where $z_{2}, z_{1}$ are such that $z_{2} \leqslant a \leqslant z_{1}, \int_{z_{2}}^{z_{1}}\left[\frac{u^{\prime}\left(W_{\Delta}-F_{X}^{-1}\left(z_{1}\right)+F_{X}^{-1}\left(z_{2}\right)\right)\left(1-T\left(z_{1}\right)\right)}{1-z_{1}}-u^{\prime}\left(W_{\Delta}-F_{X}^{-1}(t)+\right.\right.$ $\left.\left.F_{X}^{-1}\left(z_{2}\right)\right) T^{\prime}(t)\right] d t=0$ and $\int_{0}^{1} G^{*}(z) d z=\Delta$.

PROOF: The conclusion is a direct consequence of Lemma 5.3.

Note that any pair $\left(z_{2}, z_{1}\right)$ satisfying the requirements in Proposition 5.2 leads to an optimal solution to (66). Therefore such a pair $\left(z_{2}, z_{1}\right)$ is unique as the optimal solution to (66) is unique.

Proposition 5.1 and Proposition 5.2 give two qualitatively distinct optimal contracts for any given $0<\Delta<E[X]$, and the two cases are divided depending on whether or not $\Delta<K_{\Delta}$. However, $K_{\Delta}$ in general depends on $\Delta$ in an implicit and complicated way; so it is hard to compare $\Delta$ and $K_{\Delta}$. Nevertheless we are able to treat at least two cases where $A_{u}(z)$ is either a constant or strictly decreasing in $z$.

First, assume that the utility function exhibits constant absolute risk aversion, e.g. $u(z)=1-e^{-\alpha z}$ $\forall z \in \mathbb{R}^{+}$. Then it is easy to see from (22) that $l_{\Delta}$ is independent of $\Delta$, and hence so is $K_{\Delta}$. In this case, denote $K \equiv K_{\Delta}$ and $\widehat{\pi}=(1+\rho)(E[X]-K)$. Then we have the following result.

Theorem 5.4 Assume that Assumptions 2.1, 2.3, and 5.2 hold, and that $u(\cdot)$ exhibits constant absolute risk aversion. Then the optimal indemnity function $I^{*}(\cdot)$ to Problem (2) is given as

(i) If $\pi=(1+\rho) E[X]$, then $I^{*}(z)=z$ for $z \in[0, M]$.

(ii) If $\widehat{\pi}<\pi<(1+\rho) E[X]$, then

$$
I^{*}(z)= \begin{cases}z, & \text { if } 0 \leqslant z<F_{X}^{-1}\left(z_{2}\right), \\ F_{X}^{-1}\left(z_{2}\right), & \text { if } F_{X}^{-1}\left(z_{2}\right) \leqslant z<F_{X}^{-1}\left(z_{1}\right), \\ z-F_{X}^{-1}\left(z_{1}\right)+F_{X}^{-1}\left(z_{2}\right), & \text { if } F_{X}^{-1}\left(z_{1}\right) \leqslant z \leqslant M,\end{cases}
$$

where $\left(z_{2}, z_{1}\right)$ is the unique pair satisfying $z_{2} \leqslant a \leqslant z_{1}, \int_{z_{2}}^{z_{1}}\left[\frac{u^{\prime}\left(W_{\Delta}-F_{X}^{-1}\left(z_{1}\right)+F_{X}^{-1}\left(z_{2}\right)\right)\left(1-T\left(z_{1}\right)\right)}{1-z_{1}}-\right.$ $\left.u^{\prime}\left(W_{\Delta}-F_{X}^{-1}(t)+F_{X}^{-1}\left(z_{2}\right)\right) T^{\prime}(t)\right] d t=0$, and $E\left[I^{*}(X)\right]=\frac{\pi}{1+\rho}$.

(iii) If $0 \leqslant \pi \leqslant \widehat{\pi}$, then

$$
I^{*}(z)= \begin{cases}0, & \text { if } 0 \leqslant z<F_{X}^{-1}(f), \\ z-F_{X}^{-1}(f), & \text { if } F_{X}^{-1}(f) \leqslant z \leqslant M,\end{cases}
$$

where $f$ is the unique scalar satisfying $E\left[I^{*}(X)\right]=\frac{\pi}{1+\rho}$. 
ProOF: The result follows from Propositions 5.1, 5.2 and the fact that $K_{\Delta}$ is a constant for any $0<\Delta<E[X]$

Now, we study the case in which $A_{u}(z)$ is strictly decreasing. We need the following lemma.

Lemma 5.5 If $0<\Delta_{1}<\Delta_{2}<E[X]$, then $a<l_{\Delta_{1}}<l_{\Delta_{2}}<c$.

PROOF: According to definition of $l_{\Delta_{1}}$, we have

$$
h_{\Delta_{1}}\left(l_{\Delta_{1}}\right)=\int_{0}^{l_{\Delta_{1}}}\left[\frac{u^{\prime}\left(W_{\Delta_{1}}-F_{X}^{-1}\left(l_{\Delta_{1}}\right)\right)\left(1-T\left(l_{\Delta_{1}}\right)\right)}{1-l_{\Delta_{1}}}-u^{\prime}\left(W_{\Delta_{1}}-F_{X}^{-1}(t)\right) T^{\prime}(t)\right] d t=0 .
$$

Since $W_{\Delta_{1}}<W_{\Delta_{2}}$, we have $\frac{u^{\prime}\left(W_{\Delta_{2}}-F_{X}^{-1}\left(l_{\Delta_{1}}\right)\right)}{u^{\prime}\left(W_{\Delta_{1}}-F_{X}^{-1}\left(l_{\Delta_{1}}\right)\right)}<\frac{u^{\prime}\left(W_{\Delta_{2}}-F_{X}^{-1}(t)\right)}{u^{\prime}\left(W_{\Delta_{1}}-F_{X}^{-1}(t)\right)}$ for $t \in\left[0, l_{\Delta_{1}}\right)$ by Lemma A.2 in Appendix A. Hence

$$
h_{\Delta_{2}}\left(l_{\Delta_{1}}\right)=\int_{0}^{l_{\Delta_{1}}}\left[\frac{u^{\prime}\left(W_{\Delta_{2}}-F_{X}^{-1}\left(l_{\Delta_{1}}\right)\right)\left(1-T\left(l_{\Delta_{1}}\right)\right)}{1-l_{\Delta_{1}}}-u^{\prime}\left(W_{\Delta_{2}}-F_{X}^{-1}(t)\right) T^{\prime}(t)\right] d t<0 .
$$

As a result $h_{\Delta_{2}}\left(l_{\Delta_{1}}\right)<0, h_{\Delta_{2}}(c)>0$. Since $h_{\Delta_{2}}^{\prime}(z)>0$ for $z \in\left[l_{\Delta_{1}}, c\right)$, we get $l_{\Delta_{2}} \in\left(l_{\Delta_{1}}, c\right)$.

Define $\Delta(d):=\int_{0}^{d} F_{X}^{-1}(z) d z+\int_{d}^{1} F_{X}^{-1}(d) d z=\int_{0}^{d} F_{X}^{-1}(z) d z+F_{X}^{-1}(d)(1-d)$ on $d \in[a, c]$. Then $\Delta^{\prime}(d)=(1-d)\left(F_{X}^{-1}\right)^{\prime}(d)>0$. Hence, $\Delta(\cdot)$ is a continuous and strictly increasing function. Determine $l_{\Delta(a)}$ and $l_{\Delta(c)}$ by $h_{\Delta(a)}\left(l_{\Delta(a)}\right)=0$ and $h_{\Delta(c)}\left(l_{\Delta(c)}\right)=0$, and set $\widetilde{\Delta}:=\Delta\left(l_{\Delta(a)}\right)$ and $\bar{\Delta}:=\Delta\left(l_{\Delta(c)}\right)$. Finally, define a function $g(\cdot)$ on $[a, c]$ as follows:

$$
g(z):=\int_{0}^{z}\left[\frac{u^{\prime}\left(W_{0}+(1+\rho) \Delta(z)-F_{X}^{-1}(z)\right)(1-T(z))}{1-z}-u^{\prime}\left(W_{0}+(1+\rho) \Delta(z)-F_{X}^{-1}(t)\right) T^{\prime}(t)\right] d t .
$$

Proposition 5.3 Assume that Assumptions 2.1, 2.3, and 5.2 hold and that $A_{u}(\cdot)$ is strictly decreasing. Then the optimal solution to (6) is given as

(i) If $\Delta=0$, then $G^{*}(z)=0 \forall 0 \leqslant z \leqslant 1$.

(ii) If $0<\Delta \leqslant \widetilde{\Delta}$, then

$$
G^{*}(z)= \begin{cases}0, & \text { if } 0 \leqslant z<z_{2}, \\ F_{X}^{-1}(z)-F_{X}^{-1}\left(z_{2}\right), & \text { if } z_{2} \leqslant z<z_{1}, \\ F_{X}^{-1}\left(z_{1}\right)-F_{X}^{-1}\left(z_{2}\right), & \text { if } z_{1} \leqslant z \leqslant 1,\end{cases}
$$

where $z_{2}, z_{1}$ are such that $z_{2} \leqslant a \leqslant z_{1}, \int_{z_{2}}^{z_{1}}\left[\frac{u^{\prime}\left(W_{\Delta}-F_{X}^{-1}\left(z_{1}\right)+F_{X}^{-1}\left(z_{2}\right)\right)\left(1-T\left(z_{1}\right)\right)}{1-z_{1}}-u^{\prime}\left(W_{\Delta}-F_{X}^{-1}(t)+\right.\right.$ $\left.\left.F_{X}^{-1}\left(z_{2}\right)\right) T^{\prime}(t)\right] d t=0$, and $\int_{0}^{1} G^{*}(z) d z=\Delta$. 
(iii) If $\widetilde{\Delta}<\Delta<\bar{\Delta}$, then let $p \in\left(l_{\Delta(a)}, l_{\Delta(c)}\right)$ such that $\Delta(p)=\Delta$. If $g(p)<0$, then

$$
G^{*}(z)= \begin{cases}0, & \text { if } 0 \leqslant z<z_{2}, \\ F_{X}^{-1}(z)-F_{X}^{-1}\left(z_{2}\right), & \text { if } z_{2} \leqslant z<z_{1}, \\ F_{X}^{-1}\left(z_{1}\right)-F_{X}^{-1}\left(z_{2}\right), & \text { if } z_{1} \leqslant z \leqslant 1\end{cases}
$$

where $z_{2}, z_{1}$ are such that $z_{2} \leqslant a \leqslant z_{1}, \int_{z_{2}}^{z_{1}}\left[\frac{u^{\prime}\left(W_{\Delta}-F_{X}^{-1}\left(z_{1}\right)+F_{X}^{-1}\left(z_{2}\right)\right)\left(1-T\left(z_{1}\right)\right)}{1-z_{1}}-u^{\prime}\left(W_{\Delta}-F_{X}^{-1}(t)+\right.\right.$ $\left.\left.F_{X}^{-1}\left(z_{2}\right)\right) T^{\prime}(t)\right] d t=0$, and $\int_{0}^{1} G^{*}(z) d z=\Delta$. If $g(p) \geqslant 0$, then

$$
G^{*}(z)= \begin{cases}F_{X}^{-1}(z), & \text { if } 0 \leqslant z<f, \\ F_{X}^{-1}(f), & \text { if } f \leqslant z \leqslant 1,\end{cases}
$$

where $f$ is such that $f<l_{\Delta(c)}$ and $\int_{0}^{1} G^{*}(z) d z=\Delta$.

(iv) If $\bar{\Delta} \leqslant \Delta \leqslant E[X]$, then

$$
G^{*}(z)= \begin{cases}F_{X}^{-1}(z), & \text { if } 0 \leqslant z<f, \\ F_{X}^{-1}(f), & \text { if } f \leqslant z \leqslant 1,\end{cases}
$$

where $f$ is such that $f \geqslant l_{\Delta(c)}$ and $\int_{0}^{1} G^{*}(z) d z=\Delta$.

Proof: (i),(ii) and (iv) are direct consequences of Proposition 5.1, 5.2 and Lemma 5.5, For (iii), there is a unique $p \in\left(l_{\Delta(a)}, l_{\Delta(c)}\right)$ such that $\Delta(p)=\Delta$, which follows from the definition of $\widetilde{\Delta}, \bar{\Delta}$ and the fact that $\Delta(\cdot)$ is a continuous and strictly increasing function. If $g(p)<0$, then $h_{\Delta}(p)<0$; hence $l_{\Delta}>p$. Therefore, $\Delta<K_{\Delta}$. The desired result follows from Proposition 5.2. The proof for $g(p) \geqslant 0$ is similar.

Let us give the result in terms of premium and indemnity function.

Theorem 5.6 Assume that Assumptions [2.1, 2.3, and 5.2 hold, and that $A_{u}(\cdot)$ is strictly decreasing. Then the optimal indemnity function $I^{*}(\cdot)$ to Problem (1) is given as

(i) If $\pi=(1+\rho) E[X]$, then $I^{*}(z)=z \forall z \in[0, M]$.

(ii) If $(1+\rho)(E[X]-\widetilde{\Delta}) \leqslant \pi<(1+\rho) E[X]$, then

$$
I^{*}(z)= \begin{cases}z, & \text { if } 0 \leqslant z<F_{X}^{-1}\left(z_{2}\right), \\ F_{X}^{-1}\left(z_{2}\right), & \text { if } F_{X}^{-1}\left(z_{2}\right) \leqslant z<F_{X}^{-1}\left(z_{1}\right), \\ z-F_{X}^{-1}\left(z_{1}\right)+F_{X}^{-1}\left(z_{2}\right), & \text { if } F_{X}^{-1}\left(z_{1}\right) \leqslant z \leqslant M,\end{cases}
$$


where $\left(z_{2}, z_{1}\right)$ is the unique pair satisfying $z_{2} \leqslant a \leqslant z_{1}, \int_{z_{2}}^{z_{1}}\left[\frac{u^{\prime}\left(W_{\Delta}-F_{X}^{-1}\left(z_{1}\right)+F_{X}^{-1}\left(z_{2}\right)\right)\left(1-T\left(z_{1}\right)\right)}{1-z_{1}}-\right.$ $\left.u^{\prime}\left(W_{\Delta}-F_{X}^{-1}(t)+F_{X}^{-1}\left(z_{2}\right)\right) T^{\prime}(t)\right] d t=0$, and $E\left[I^{*}(X)\right]=\frac{\pi}{1+\rho}$.

(iii) If $(1+\rho)(E[X]-\bar{\Delta})<\pi<(1+\rho)(E[X]-\widetilde{\Delta})$, then let $p \in\left(l_{\Delta(a)}, l_{\Delta(c)}\right)$ such that $\Delta(p)=$ $E[X]-\frac{\pi}{1+\rho}$. If $g(p)<0$, then

$$
I^{*}(z)= \begin{cases}z, & \text { if } 0 \leqslant z<F_{X}^{-1}\left(z_{2}\right), \\ F_{X}^{-1}\left(z_{2}\right), & \text { if } F_{X}^{-1}\left(z_{2}\right) \leqslant z<F_{X}^{-1}\left(z_{1}\right), \\ z-F_{X}^{-1}\left(z_{1}\right)+F_{X}^{-1}\left(z_{2}\right), & \text { if } F_{X}^{-1}\left(z_{1}\right) \leqslant z \leqslant M,\end{cases}
$$

where $\left(z_{2}, z_{1}\right)$ is the unique pair satisfying $z_{2} \leqslant a \leqslant z_{1}, \int_{z_{2}}^{z_{1}}\left[\frac{u^{\prime}\left(W_{\Delta}-F_{X}^{-1}\left(z_{1}\right)+F_{X}^{-1}\left(z_{2}\right)\right)\left(1-T\left(z_{1}\right)\right)}{1-z_{1}}-\right.$ $\left.u^{\prime}\left(W_{\Delta}-F_{X}^{-1}(t)+F_{X}^{-1}\left(z_{2}\right)\right) T^{\prime}(t)\right] d t=0$, and $E\left[I^{*}(X)\right]=\frac{\pi}{1+\rho}$. If $g(p) \geqslant 0$, then

$$
I^{*}(z)= \begin{cases}0, & \text { if } 0 \leqslant z<F_{X}^{-1}(f), \\ z-F_{X}^{-1}(f), & \text { if } F_{X}^{-1}(f) \leqslant z \leqslant M,\end{cases}
$$

where $q$ is the unique number satisfying $f<l_{\Delta(c)}$ and $E\left[I^{*}(X)\right]=\frac{\pi}{1+\rho}$.

(iv) If $0 \leqslant \pi \leqslant(1+\rho)(E[X]-\bar{\Delta})$, then

$$
I^{*}(z)= \begin{cases}0, & \text { if } 0 \leqslant z<F_{X}^{-1}(f), \\ z-F_{X}^{-1}(f), & \text { if } F_{X}^{-1}(f) \leqslant z \leqslant M,\end{cases}
$$

where $q$ is the unique number satisfying $f \geqslant l_{\Delta(c)}$ and $E\left[I^{*}(X)\right]=\frac{\pi}{1+\rho}$.

PRoOF: It follows easily from Proposition 5.3.

\section{$6 \quad$ Numerical Illustrations}

In this section, we use a numerical example to illustrate our result with a given premium $\pi$. we take the same numerical setting as in Bernard et al. (2015) for a comparison purpose: The loss $X$ follows a truncated exponential distribution with the density function $f(x)=\frac{m e^{-m x}}{1-e^{-m M}}$, where the intensity parameter $m=0.1$, and $M=10$. The initial wealth $W_{0}=15$, and $u(x)=1-e^{-\gamma x}$ with $\gamma=0.02$. Moreover, $\rho=0.2$ and $\pi=3$. Finally, the weighting function $T_{\theta}(x)=\frac{x^{\theta}}{\left(x^{\theta}+(1-x)^{\theta}\right)^{\frac{1}{\theta}}}$ with $\theta=0.5$. We can verify that the assumptions of Theorem 5.4 is satisfied under this setting. 


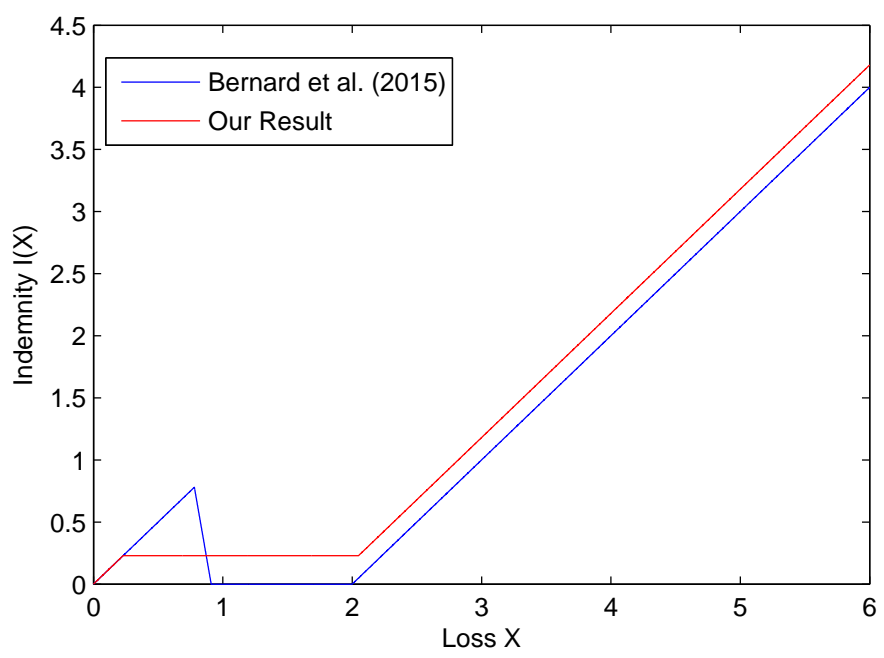

Figure 3: Our result vs Bernard et al. (2015).

The optimal indemnity obtained by Bernard et al. (2015) is plotted in blue in Figure 3. We note that in the result of Bernard et al. (2015), if the loss is between 1 and 2, then the insured has the incentive to hide part of the loss in order to be paid with a larger compensation. By contrast, our indemnity function, depicted in red, is increasing and any increment in compensations is always less than or equal to the increment in losses. It effectively rules out the aforementioned behavior of moral hazard.

\section{Conclusion}

In this paper, we have studied an optimal insurance design problem where the insured has the RDU preference. There are documented evidences proving that this preference captures human behaviors better than the EU preference. The main contribution of our work is that our optimal contracts are monotone with respect to losses, thereby eliminating the potential problem of moral hazard associated with the existing results.

An interesting conclusion from our results is that, under our assumptions (in particular Assumption 5.2 (ii)), there are only two types of non-trivial optimal contracts possible, one being the classical deductible and the other the threefold contract covering both small and large losses.

While we have demonstrated that Assumption 5.2-(ii) holds for many economically interesting cases, removing this assumption remains a mathematically outstanding open problem. 


\section{A Some Lemmas}

In this part, we prove some lemmas which have been used in Section 5 .

Lemma A.1 Assume $T(\cdot):[0,1] \mapsto[0,1]$ satisfies Assumption 2.3. We have the following results:

(i) If $a<z$, then $T^{\prime}(z)<\frac{1-T(z)}{1-z}$.

(ii) If $a<z_{2}<b$ and $z_{2}<z_{1}<1$, then $\frac{1-T\left(z_{1}\right)}{1-z_{1}}>\frac{T\left(z_{1}\right)-T\left(z_{2}\right)}{z_{1}-z_{2}}$.

ProOF: (i) If $a<z \leqslant b$, then $T^{\prime}(z)<T^{\prime}(a)<\frac{1-T(z)}{1-z}$. If $b<z$, then $T^{\prime}(z)<\frac{1-T(z)}{1-z}$ since $T(\cdot)$ is convex and strictly increasing on $[b, 1]$.

(ii) Since $1-T\left(z_{1}\right), 1-z_{1}, T\left(z_{1}\right)-T\left(z_{2}\right)$, and $z_{1}-z_{2}$ are all strictly positive, we have

$$
\begin{aligned}
\frac{1-T\left(z_{1}\right)}{1-z_{1}}>\frac{T\left(z_{1}\right)-T\left(z_{2}\right)}{z_{1}-z_{2}} & \Longleftrightarrow \frac{1-T\left(z_{1}\right)}{1-z_{1}}>\frac{\left(1-T\left(z_{1}\right)\right)+\left(T\left(z_{1}\right)-T\left(z_{2}\right)\right)}{\left(1-z_{1}\right)+\left(z_{1}-z_{2}\right)} \\
& \Longleftrightarrow \frac{1-T\left(z_{1}\right)}{1-z_{1}}>\frac{1-T\left(z_{2}\right)}{1-z_{2}} .
\end{aligned}
$$

However, $\frac{1-T\left(z_{1}\right)}{1-z_{1}}>\frac{1-T\left(z_{2}\right)}{1-z_{2}}$ follows from Lemma 4.1.

For fixed $x>0$, define $q(z):=u^{\prime}(x+z) u^{\prime}(x-z)$ on $z \in(0, x)$.

Lemma A.2 If $-\frac{u^{\prime \prime}(z)}{u^{\prime}(z)}$ is strictly decreasing, then $q(z)$ is a strictly increasing function on $z \in(0, x)$.

Proof: We take derivative:

$$
\begin{aligned}
q^{\prime}(z) & =u^{\prime \prime}(x+z) u^{\prime}(x-z)-u^{\prime}(x+z) u^{\prime \prime}(x-z) \\
& =u^{\prime}(x+z) u^{\prime}(x-z)\left[\left(-\frac{u^{\prime \prime}(x-z)}{u^{\prime}(x-z)}\right)-\left(-\frac{u^{\prime \prime}(x+z)}{u^{\prime}(x+z)}\right)\right]>0 .
\end{aligned}
$$

Hence, we get the result.

\section{B Existence of Optimal Solutions to (6) and (8)}

We first prove that the constraint set $\mathbb{G}$ is compact under some norm. We consider all the continuous functions on $[0,1]$, denoted as $C[0,1]$. Define a metric between $x(\cdot), y(\cdot)$ as $\rho(x(\cdot), y(\cdot))=$ $\max _{0 \leqslant t \leqslant 1}|x(t)-y(t)|, \forall x(\cdot), y(\cdot) \in C[0,1]$. Clearly, $C[0,1]$ is a metric space under $\rho$. By ArzelaAscoli's theorem, for any sequence $\left(G_{n}(\cdot)\right)_{n \in \mathbb{N}}$ in $\mathbb{G}$, there exists a subsequence $G_{n_{k}}(\cdot)$ that converges in $C[0,1]$ under $\rho$. 
Lemma B.1 The feasible set $\mathbb{G}$ is compact under $\rho$.

ProOF: For any sequence $\left(G_{n}(\cdot)\right)_{n \in \mathbb{N}}$ in $\mathbb{G}$, there exists a subsequence $G_{n_{k}}(\cdot)$ that uniformly converges in $G^{*}(\cdot) \in C[0,1]$. We now prove that $G^{*}(\cdot) \in \mathbb{G}$. If there exist $a>b$ such that $G^{*}(b)-G^{*}(a)=\eta>0$, then take $\varepsilon:=\frac{1}{3} \eta$. If follows from the uniform convergence that there exists $K$ such that $\rho\left(G_{n_{k}}(\cdot), G^{*}(\cdot)\right) \leqslant \varepsilon \forall k \geqslant K$. Hence, $0<\eta=G^{*}(b)-G^{*}(a)=G^{*}(b)-G_{n_{k}}(b)+$ $G_{n_{k}}(b)-G_{n_{k}}(a)+G_{n_{k}}(a)-G^{*}(a) \leqslant \varepsilon+0+\varepsilon=\frac{2}{3} \eta \forall k \geqslant K$, which is a contradiction. This proves that $0 \leqslant G^{*}(a)-G^{*}(b) \forall a>b$. Similarly, we can prove that $G^{*}(a)-G^{*}(b) \leqslant F_{X}^{-1}(a)-F_{X}^{-1}(b)$.

The existence of optimal solutions to (6) and (8) can be established now. For example, for (8) let $v_{\lambda}(\Delta)$ be the optimal value of (8) under given $\lambda$ and $\Delta$. We can take a sequence $\left(G_{n}(\cdot)\right)_{n \in \mathbb{N}}$ in $\mathbb{G}$ such that $v_{\lambda}(\Delta)=\lim _{n \uparrow+\infty} U_{\Delta}\left(\lambda, G_{n}(\cdot)\right)$. Then, according to Lemma B.1, there exists a subsequence $G_{n_{k}}(\cdot)$ converging to $G^{*}(\cdot)$ in $\mathbb{G}$ and $G^{*}(\cdot)$ is optimal solution to (8) . For (6) , the proof is similar.

\section{Existence of Lagrangian Multiplier to (6)}

For the following lemma, refer to Komiya (1988) for an elementary proof.

Lemma C.1 (Sion's Minimax Theorem) Let X be a compact convex subset of a linear topological space and $Y$ a convex subset of a linear topological space. If $f$ is a real-valued function on $X \times Y$ such that $f(x, \cdot)$ is continuous and concave on $Y \forall x \in X$, and $f(\cdot, y)$ is continuous and convex on $X \forall y \in Y$, then, $\min _{x \in X} \max _{y \in Y} f(x, y)=\max _{y \in Y} \min _{x \in X} f(x, y)$.

Proposition C.1 For any $0<\Delta<E[X]$, there is $\lambda^{*}$ such that $\widetilde{G}_{\lambda^{*}}(\cdot)$ is optimal solution to (8) under $\lambda^{*}$ and $\int_{0}^{1} \widetilde{G}_{\lambda^{*}}(z) d z=\Delta$.

ProOf: Let $\Delta$ be given with $0<\Delta<E[X]$. Denote by $G^{*}(\cdot)$ the optimal solution to (66) under $\Delta$ (it is easy to show $\int_{0}^{1} G^{*}(z) d z=\Delta$ ) and by $\widetilde{G}_{\lambda}(\cdot)$ the optimal solution to (8) under $\lambda$ and $\Delta$. Denote by $v(\Delta)$ and $v(\lambda, \Delta)$ be respectively the optimal values of (6) and (8) .

We first prove that $v(\lambda, \Delta)$ is a convex function in $\lambda$ for given $\Delta$. Noting that $U_{\Delta}(\lambda, G(\cdot))$ is linear in $\lambda$ for any given $G(\cdot)$, we have

$$
\begin{aligned}
v\left(\alpha \lambda_{1}+(1-\alpha) \lambda_{2}, \Delta\right) & =\max _{G(\cdot)} U_{\Delta}\left(\alpha \lambda_{1}+(1-\alpha) \lambda_{2}, G(\cdot)\right) \\
& =\max _{G(\cdot)}\left\{\alpha U_{\Delta}\left(\lambda_{1}, G(\cdot)\right)+(1-\alpha) U_{\Delta}\left(\lambda_{2}, G(\cdot)\right)\right\}
\end{aligned}
$$




$$
\begin{aligned}
& \leqslant \max _{G(\cdot)}\left\{\alpha U_{\Delta}\left(\lambda_{1}, G(\cdot)\right)\right\}+\max _{G(\cdot)}\left\{(1-\alpha) U_{\Delta}\left(\lambda_{2}, G(\cdot)\right)\right\} \\
& =\alpha \max _{G(\cdot)}\left\{U_{\Delta}\left(\lambda_{1}, G(\cdot)\right)\right\}+(1-\alpha) \max _{G(\cdot)}\left\{U_{\Delta}\left(\lambda_{2}, G(\cdot)\right)\right\} \\
& =\alpha v\left(\lambda_{1}, \Delta\right)+(1-\alpha) v\left(\lambda_{2}, \Delta\right) .
\end{aligned}
$$

Moreover, by Sion's minimax theorem, the following equality holds: $\max _{0 \leqslant \lambda} \min _{G(\cdot) \in \mathbb{G}}-U_{\Delta}(\lambda, G(\cdot))=$ $\min _{G(\cdot) \in \mathbb{G}} \max _{0 \leqslant \lambda}-U_{\Delta}(\lambda, G(\cdot))$; hence $\min _{0 \leqslant \lambda} \max _{G(\cdot) \in \mathbb{G}} U_{\Delta}(\lambda, G(\cdot))=\max _{G(\cdot) \in \mathbb{G}} \min _{0 \leqslant \lambda} U_{\Delta}(\lambda, G(\cdot))$. Finally, we have $v(\Delta)=\inf _{0 \leqslant \lambda} v(\lambda, \Delta)$ (i.e. $\left.\min _{0 \leqslant \lambda} \max _{G(\cdot) \in \mathbb{G}} U_{\Delta}(\lambda, G(\cdot))=U_{\Delta}\left(G^{*}(\cdot)\right)\right)$.

Let us denote $\bar{\lambda}:=\frac{v(\Delta)+1}{\int_{0}^{1} F_{X}^{-1}(z) d z-\Delta}=\frac{U_{\Delta}\left(G^{*}(\cdot)\right)+1}{E[X]-\Delta}$. For any $\lambda \geqslant \bar{\lambda}$, we have

$$
\begin{aligned}
v(\lambda, \Delta) & \left.=\max _{G(\cdot) \in \mathbb{G}} U_{\Delta}(\lambda, G(\cdot)) \geqslant U_{\Delta}\left(\lambda, F_{X}^{-1}(z)\right)\right) \\
& =\int_{0}^{1} u\left(W_{\Delta}-F_{X}^{-1}(z)\right) T^{\prime}(z) d z+\lambda\left(\int_{0}^{1} F_{X}^{-1}(z) d z-\Delta\right) \geqslant \lambda\left(\int_{0}^{1} F_{X}^{-1}(z) d z-\Delta\right) \\
& \geqslant \bar{\lambda}\left(\int_{0}^{1} F_{X}^{-1}(z) d z-\Delta\right)\left(\text { since } \int_{0}^{1} F_{X}^{-1}(z) d z>\Delta\right) \\
& =v(\Delta)+1,
\end{aligned}
$$

which yields $v(\Delta)=\inf _{0 \leqslant \lambda} v(\lambda, \Delta)=\inf _{0 \leqslant \lambda \leqslant \bar{\lambda}} v(\lambda, \Delta)$.

Therefore, by using the convexity of $v(\lambda, \Delta)$, we can find the optimal $\lambda^{*} \in[0, \bar{\lambda}]$ minimizes the right part, and satisfies that $v(\Delta)=v\left(\lambda^{*}, \Delta\right)$. Moreover,

$$
\begin{aligned}
v\left(\lambda^{*}, \Delta\right) & \geqslant U_{\Delta}\left(\lambda^{*}, G^{*}(\cdot)\right)=\int_{0}^{1} u\left(W_{\Delta}-G^{*}(z)\right) T^{\prime}(z) d z+\lambda^{*}\left(\int_{0}^{1} G^{*}(z) d z-\Delta\right) \\
& =\int_{0}^{1}\left[u\left(W_{\Delta}-G^{*}(z)\right) T^{\prime}(z)\right] d z=U_{\Delta}\left(G^{*}(\cdot)\right)=v(\Delta) .
\end{aligned}
$$

The second equality comes from the fact that $G^{*}(\cdot)$ is the optimal solution to (66) under $\Delta$; hence $\int_{0}^{1} G^{*}(z) d z=\Delta$. By $v(\Delta)=v\left(\lambda^{*}, \Delta\right)$ and $v\left(\lambda^{*}, \Delta\right) \geqslant U_{\Delta}\left(\lambda^{*}, G^{*}(\cdot)\right)=v(\Delta)$, we have $G^{*}(\cdot)$ is optimal solution to (8) under given $\lambda^{*}$. And, by uniqueness of optimal solutions to (8) , we know that $G^{*}(\cdot)$ is the unique optimal solution to (8) under given $\lambda^{*}$ and satisfying $\int_{0}^{1} G^{*}(z) d z=\Delta$. 


\section{References}

[1] Arrow, K.J. (1963): Uncertainty and the welfare economics of medical care, The American Economic Review, Vol. 53(5), pp. 941-973

[2] Arrow, K.J. (1971): Essays in the theory of risk-bearing, North-Holland Publishing Company, Amsterdam and London

[3] Barseghyan, L., Molinari, F., O’Donoghue, T., and Teitelbaum, J.C. (2013): The nature of risk preferences: evidence from insurance choices, The American Economic Review , Vol. 103(6), pp. 2499-2529

[4] Bernard, C., He, X.D., Yan, J.-A., And Zhou, X.Y. (2015): Optimal insurance design under rank-dependent expected utility, Mathematical Finance, Vol. 25, pp. 154-186

[5] Carlier, G., And Dana, R.-A. (2008): Two-persons efficient risk-sharing and equilibria for concave law-invariant utilities, Economic Theory, Vol. 36(2), pp. 189-223

[6] Chateauneuf, A., Dana, R.-A., and Tallon. J.-M. (2000): Optimal risk-sharing rules and equilibria with choquet-expected-utility, Journal of Mathematical Economics, Vol. 34(2), pp. 191-214

[7] Dana, R.-A., And Scarsini, M. (2007): Optimal risk sharing with background risk, Journal of Economic Theory, Vol. 133(1), pp. 152-176

[8] Gollier, C., And Schlesinger, H. (1996): Arrow's theorem on the optimality of deductibles: a stochastic dominance approach, Economic Theory, Vol. 7(2), pp. 359-363

[9] He, X.D., And Zhou, X.Y. (2011): Portfolio choice via quantiles, Mathematical Finance, Vol. 21(2), pp. 203-231

[10] Jin, H., And Zhou, X.Y. (2008): Behavioral portfolio selection in continuous time, Mathematical Finance, Vol. 18(3), pp. 385-426

[11] Komiya, H. (1988): Elementary proof for sion's minimax theorem, Kodai Mathematical Journal , Vol. 11(1), pp. 5-7

[12] Quigain (1982): A theory of anticipated utility, Journal of Economic and Behavioral Organization, Vol. 3(4), pp. 323-343 
[13] Raviv, A. (1979): The design of an optimal insurance policy, The American Economic Review , Vol. 69(1), pp. 84-96

[14] Tversky, A., And Kahneman, D. (1992): Advances in prospect theory: Cumulative representation of uncertainty, Journal of Risk and Uncertainty, Vol. 5(4), pp. 297-323

[15] YAARI, M.E. (1987): The dual theory of choice under risk, Econometrica, Vol. 55(1), pp. 95-115 